\title{
VARIATIONAL PROBLEMS WITH TWO PHASES AND THEIR FREE BOUNDARIES \\ BY
}

\author{
HANS WILHELM ALT, LUIS A. CAFFARELLI AND AVNER FRIEDMAN ${ }^{1}$
}

ABSTRACT. The problem of minimizing $\int\left[\left.\nabla v\right|^{2}+q^{2}(x) \lambda^{2}(v)\right] d x$ in an appropriate class of functions $v$ is considered. Here $q(x) \neq 0$ and $\lambda^{2}(v)=\lambda_{1}^{2}$ if $v<0$, = $\lambda_{2}^{2}$ if $v>0$. Any minimizer $u$ is harmonic in $\{u \neq 0\}$ and $|\nabla u|^{2}$ has a jump

$$
q^{2}(x)\left(\lambda_{1}^{2}-\lambda_{2}^{2}\right)
$$

across the free boundary $\{u \neq 0\}$. Regularity and various properties are established for the minimizer $u$ and for the free boundary.

Introduction. In this paper we consider the problem of minimizing

$$
J(v)=\int_{\Omega}\left[|\nabla v|^{2}+q^{2}(x) \lambda^{2}(v)\right] d x, \quad v \in K,
$$

where $q^{2}(x) \neq 0$,

$$
\lambda^{2}(v)= \begin{cases}\lambda_{1}^{2} & \text { if } v<0 \\ \lambda_{2}^{2} & \text { if } v>0\end{cases}
$$

and $\lambda^{2}(v)$ is lower semicontinuous at $v=0$; it is assumed that $\lambda_{i}^{2}>0$ and $\Lambda=\lambda_{1}^{2}-$ $\lambda_{2}^{2} \neq 0$. The class $K$ consists of all functions $v$ in $L_{\text {loc }}^{1}(\Omega)$, with $\nabla v \in L^{2}(\Omega)$ such that $v=u^{0}$ on a given open subset $S$ of $\partial \Omega$, and $\Omega$ is a domain in $R^{n}$.

The analogous problem for functions in $K^{+}=\{v \in K, v \geqslant 0$ a.e. $\}$ was studied in [1]; in that paper it was proved that any (local) minimizer of $J(v)$ in $K^{+}$is Lipschitz continuous and, if $n=2$, the free boundary $\partial\{u>0\}$ is analytic if $q(x)$ is analytic.

The present variational problem is motivated by applications to the flow of two liquids in models of jets and cavities; these applications will be studied in other forthcoming papers $[\mathbf{5}, \mathbf{6}]$. The present work is aimed at extending results of [1]. In particular, we shall establish nondegeneracy theorems, the Lipschitz continuity of the solution, and some properties of the free boundary; for $n=2$ the free boundary is proved to be continuously differentiable.

A new and rather powerful tool introduced in this paper is the monotonicity formula (Lemma 5.1) asserting that, for a minimizer $u$, if $u\left(x_{0}\right)=0$ then

$$
r^{-4} \int_{B_{r}\left(x_{0}\right)} \rho^{2-n}\left|\nabla u^{+}\right|^{2} d x \cdot \int_{B_{r}\left(x_{0}\right)} \rho^{2-n}\left|\nabla u^{-}\right|^{2} d x \nearrow \quad \text { if } r \nearrow
$$

Received by the editors December 10, 1982.

1980 Mathematics Subject Classification. Primary 35A15, 35J65, 49B22; Secondary 76B10.

'This work is partially supported by Deutsche Forschungsgemeinschaft, SFB 72 and by National Science Foundation Grants 7406375 A01 and MCS7915171. 
This is used in establishing Lipschitz continuity and in identifying blow-up limits. The differentiability of the free boundary for $n=2$ also involves a new set of ideas, exploiting among other things, the monotonicity formula.

1. Existence. Let $\Omega$ be a domain in $R^{n}$ with boundary $\partial \Omega$ which is locally a Lipschitz graph. Let $S$ be a nonempty open subset of $\partial \Omega$ and let $u^{0}$ be a given function in $L_{\mathrm{loc}}^{1}(\Omega)$ with $\nabla u^{0} \in L^{2}(\Omega)$. Let $q(x)$ be a strictly positive uniformly Lipschitz continuous function in compact subsets of $\bar{\Omega}$, and let $\lambda(u)$ be the function

$$
\lambda(u)= \begin{cases}\lambda_{1} & \text { if } u<0, \\ \lambda_{2} & \text { if } u>0\end{cases}
$$

where $\lambda_{1}, \lambda_{2} \geqslant 0$, and define $\lambda(0)$ such that

$$
0 \leqslant \lambda(0) \leqslant \min \left\{\lambda_{1}, \lambda_{2}\right\}
$$

We assume that

$$
\Lambda=\lambda_{1}^{2}-\lambda_{2}^{2} \neq 0 .
$$

Finally, set $Q(u, x)=q(x) \lambda(u)$.

We introduce the convex set

$$
K=\left\{v \in L_{\mathrm{loc}}^{1}(\Omega), \nabla v \in L^{2}(\Omega), v=u^{0} \text { on } S\right\}
$$

and the functional

$$
J(v)=\int_{\Omega}\left(|\nabla v|^{2}+Q^{2}(v, x)\right) d x, \quad v \in K .
$$

Problem (J). Find $u \in K$ such that $J(u)=\min _{v \in K} J(v)$.

TheOREM 1.1. If $J\left(u^{0}\right)<\infty$ then there exists a solution of Problem (J).

Proof. Take a minimizing sequence $u_{k}$. Then the $\nabla u_{k}$ are uniformly bounded in $L^{2}(\Omega)$. Since $u_{k}-u^{0}=0$ on $S, S$ open and nonempty, we can estimate $u_{k}-u^{0}$ in $L^{2}\left(\Omega \cap B_{R}\right)$ for any ball $B_{R}=\{|x|<R\}$ and deduce that, for a subsequence,

$$
\begin{aligned}
\nabla u_{k} & \rightarrow \nabla u \quad \text { weakly in } L_{\text {loc }}^{2}(\Omega), \\
u_{k} & \rightarrow u \quad \text { a.e. in } \Omega, \\
Q^{2}\left(u_{k}, x\right) & \rightarrow \gamma \quad \text { weakly star in } L_{\text {loc }}^{\infty}(\Omega),
\end{aligned}
$$

where $\gamma=Q^{2}(u, x)$ if $u \neq 0$, and $\gamma \geqslant Q^{2}(u, x)$ if $u=0$ (by (1.2)). Hence,

$$
\begin{aligned}
\int_{\Omega \cap B_{R}}\left(|\nabla u|^{2}+Q^{2}(u, x)\right) & \leqslant \liminf _{k \rightarrow \infty} \int_{\Omega \cap B_{R}}\left|\nabla u_{k}\right|^{2}+\lim _{k \rightarrow \infty} \int_{\Omega \cap B_{R}} Q^{2}\left(u_{k}, x\right) \\
& \leqslant \liminf _{k \rightarrow \infty} J\left(u_{k}\right) .
\end{aligned}
$$

Letting $R \rightarrow \infty$ we see that $u$ is an absolute minimum for $J$. 
2. Continuity, subharmonicity and the free boundary condition. We denote a solution of Problem (J) by $u$.

THEGREM 2.1. For any compact subset $D$ of $\Omega$ there exists a constant $C$ such that

$$
|u(x)-u(y)| \leqslant C|x-y| \log (1 /|x-y|)
$$

if $x, y \in D,|x-y|<\frac{1}{2}$.

Proof. Let $B_{r}$ be any ball of radius $r$ in $D$ and denote by $v_{r}$ the solution of

$$
\nabla v_{r}=0 \quad \text { in } B_{r}, \quad v_{r}=u \text { on } \partial B_{r} .
$$

Then, by the minimality of $u$,

$$
\int_{B_{r}}\left(|\nabla u|^{2}+Q^{2}(u, x)\right) \leqslant \int_{B_{r}}\left(\left|\nabla v_{r}\right|^{2}+Q^{2}\left(v_{r}, x\right)\right) .
$$

It follows that $\int_{B_{r}}\left(|\nabla u|^{2}-\left|\nabla v_{r}\right|^{2}\right) \leqslant C r^{n}$. But the left-hand side is equal to

$$
\int_{B_{r}} \nabla\left(u-v_{r}\right) \cdot \nabla\left(u+v_{r}\right)=\int_{B_{r}}\left|\nabla\left(u-v_{r}\right)\right|^{2}+2 \int_{B_{r}} \nabla\left(u-v_{r}\right) \cdot \nabla v_{r}
$$

and the last integral vanishes, by (2.1). Consequently, $\int_{B_{r}}\left|\nabla\left(u-v_{r}\right)\right|^{2} \leqslant C r^{n}$.

Proceeding as in [11, Theorem, 5.3.6], one can establish that

$$
\int_{B_{r}}\left|\nabla\left(u-v_{r}\right)\right|^{2} \leqslant C(R) r^{n}(\log R / r+1) \quad \text { if } 0<r<R,
$$

so that

$$
\int_{B_{r}}|\nabla u|^{2} \leqslant C(R) r^{n}\left(\log \frac{R}{r}+1\right),
$$

from which the assertion follows as in [11, Theorem 3.5.2].

THEOREM 2.2. The function $u$ is harmonic in $\{u \neq 0\}$.

Proof. For any $\zeta \in C_{0}^{\prime}(\Omega \backslash\{u=0\}), u \pm \varepsilon \zeta$ is in $K$ for any $\varepsilon>0$. Hence,

$$
0=\lim _{\varepsilon \downarrow 0} \frac{1}{2 \varepsilon}(J(u+\varepsilon \zeta)-J(\zeta))=\int_{\Omega} \nabla \zeta \cdot \nabla u .
$$

THEOREM 2.3. If $\lambda(0)=\lambda_{1}$ and $\Lambda<0\left(\lambda(0)=\lambda_{2}\right.$ and $\left.\Lambda>0\right)$ then $u$ is subharmonic (superharmonic) in $\Omega$.

Proof. Defining $v$ by $(2.1), B_{r} \subset \Omega$, we have $J(u) \leqslant J(\min (u, v))$, which gives, if $\lambda(0)=\lambda_{1}$,

$$
\begin{aligned}
I & \equiv \int_{B_{r}}\left[|\nabla u|^{2}-|\nabla \min (u, v)|^{2}\right] \leqslant \int_{B_{r}}\left[Q^{2}(\min (u, v), x)-Q^{2}(u, x)\right] \\
& =\int_{B_{r} \cap\{u>v\}}\left[Q^{2}(v, x)-Q^{2}(u, x)\right]=\int_{B_{r} \cap\{u \geqslant 0>v\}} \Lambda q^{2}(x) .
\end{aligned}
$$


But

$$
\begin{aligned}
I & =\int_{B_{r}} \nabla \max (u-v, 0) \cdot \nabla(u+v) \\
& =\int_{B_{r}} \nabla \max (u-v, 0) \cdot \nabla(u-v)+2 \int_{B_{r}} \nabla \max (u-v, 0) \cdot \nabla v \\
& =\int_{B_{r}}|\nabla \max (u-v, 0)|^{2} .
\end{aligned}
$$

Hence, $\Lambda<0$ implies $u \leqslant v$, i.e., $u$ is subharmonic. Similarly, if $\lambda(0)=\lambda_{2}$ and $\Lambda>0$, then $u$ is superharmonic.

Definition 2.1. The set $\Gamma=\partial\{u>0\} \cup \partial\{u<0\}$ is called the free boundary.

The next theorem shows that $u$ satisfies, in a generalized sense, the equation

$$
\left|\nabla u^{-}\right|^{2}-\left|\nabla u^{+}\right|^{2}=\Lambda q^{2}(x) \text { on } \Gamma
$$

provided the set $\{u=0\}$ has zero measure.

TheOREM 2.4. Suppose meas $\{u=0\}=0$. Then, for any $\eta \in C_{0}^{1}\left(\Omega, R^{n}\right)$,

$$
\lim _{\varepsilon \downarrow 0} \int_{\partial\{u<-\varepsilon\}}\left(|\nabla u|^{2}-\lambda_{1}^{2} q^{2}(x)\right) \eta \cdot \nu+\lim _{\delta \downarrow 0} \int_{\partial\{u>\delta\}}\left(|\nabla u|^{2}-\lambda_{2}^{2} q^{2}(x)\right) \eta \cdot \nu=0
$$

where $\nu$ is the outward normal.

Proof. Let $\tau_{\varepsilon}(x)=x+\varepsilon \eta(x), \varepsilon \neq 0$, and define $u_{\varepsilon} \in K$ by $u_{\varepsilon}\left(\tau_{\varepsilon} x\right)=u(x)$. Then

$$
\begin{aligned}
0 \leqslant & J\left(u_{\varepsilon}\right)-J(u) \\
= & \int_{\Omega}\left\{\left[\left|\nabla u\left(D \tau_{\varepsilon}\right)^{-1}\right|^{2}+Q^{2}\left(u, \tau_{\varepsilon}(x)\right)\right] \operatorname{det}\left(D \tau_{\varepsilon}\right)-\left(|\nabla u|^{2}+Q^{2}(u, x)\right)\right\} \\
= & \varepsilon \int_{\Omega}\left[|\nabla u|^{2}+Q^{2}(u, x)\right] \nabla \cdot \eta \\
& +\varepsilon \int_{\Omega}\left[-2 \nabla u D \eta \nabla u+\nabla_{x} Q^{2}(u, x) \cdot \eta\right]+O(\varepsilon) .
\end{aligned}
$$

The linear term in $\varepsilon$ must vanish, giving (since $\Delta u=0$ in $\{u \neq 0\}$ )

$$
\begin{aligned}
0= & \lim _{\varepsilon \downarrow 0, \delta \downarrow 0} \int_{\Omega \backslash\{-\varepsilon<u<\delta\}} \nabla \cdot\left[\left(|\nabla u|^{2}+Q^{2}(u, x)\right) \eta-2 \eta \cdot \nabla u \nabla u\right] \\
= & \lim _{\varepsilon \downarrow 0} \int_{\partial\{u<-\varepsilon\}}\left[\left(|\nabla u|^{2}+Q^{2}(u, x)\right) \eta-2 \eta \cdot \nabla u \nabla u\right] \cdot \nu \\
& +\lim _{\delta \downarrow 0} \int_{\partial\{u>\delta\}}\left[\left(|\nabla u|^{2}+Q^{2}(u, x)\right) \eta-2 \eta \cdot \nabla u \nabla u\right] \cdot \nu \\
= & \lim _{\varepsilon \downarrow 0} \int_{\partial\{u<-\varepsilon\}}\left[\lambda_{1}^{2} q^{2}(x)-|\nabla u|^{2}\right] \eta \cdot \nu \\
& +\lim _{\delta \downarrow 0} \int_{\partial\{u>\delta\}}\left[\lambda_{2}^{2} q^{2}(x)-|\nabla u|^{2}\right] \eta \cdot \nu .
\end{aligned}
$$


REMARK 2.1. If meas $\{u=0\}>0$ and if $\{u=0\}$ is a limit of increasing open sets $D_{\rho}(\rho \downarrow 0)$, then on the left-hand side of (2.2) there appears the additional term

$$
\lim _{\rho \downarrow 0} \int_{\partial D_{\rho}}\left(|\nabla u|^{2}-\lambda^{2}(0) q^{2}(x)\right) \eta \cdot \nu
$$

3. Nondegeneracy. For any function $v$ and a ball $B_{r}=B_{r}\left(x^{0}\right)$ with center $x^{0}$ and radius $r$, we set

$$
f_{\partial B_{r}} v=\frac{1}{\left|\partial B_{r}\right|} \int_{\partial B_{r}} v, \quad\left|\partial B_{r}\right|=\text { surface area of } \partial B_{r} .
$$

Let

$$
0 \leqslant q_{1} \leqslant q(x) \leqslant q_{2}<\infty, \quad|\Lambda| \geqslant l_{0}>0 .
$$

TheOrem 3.1. Suppose $\Lambda<0$. For any $0<\kappa<1$ there is a positive constant $c$ depending only on $\kappa$ and $q_{1}^{2} l_{0}$ such that if $B_{r} \subset \Omega$ and $\frac{1}{r} f_{\partial B_{r}} u^{+}<c$, then $u^{+}=0$ in $B_{\kappa r}$.

Proof. Set $\gamma=\frac{1}{r} f_{\partial B_{r}} u^{+}$. The idea of this proof is to replace $u$ in $B_{r}$ by a function $v$ satisfying

$$
\begin{aligned}
v=0 & \text { on } \partial B_{r}, \\
v=u & \text { in } B_{r} \cap\{u \leqslant 0\}, \\
v=0 & \text { in } B_{\kappa r} \cap\{u>0\}, \\
\Delta v=0 & \text { in }\left(B_{r} \backslash B_{\kappa r}\right) \cap\{u>0\}
\end{aligned}
$$

and show that $J(v)<J(u)$ if $\gamma$ is sufficiently small.

For almost any $\varepsilon>0$ the surface $\{u=\varepsilon\}$ is smooth. Choose any such small $\varepsilon$ and consider the function $v_{\varepsilon}$ satisfying

$$
\begin{aligned}
v_{\varepsilon}=u & \text { on } \partial B_{r}, \\
v_{\varepsilon}=u & \text { in } B_{r} \cap\{u<\varepsilon\}, \\
v_{\varepsilon}=\varepsilon & \text { in } B_{\kappa r} \cap\{u>\varepsilon\}, \\
\Delta v_{\varepsilon}=0 & \text { in } D_{\varepsilon}^{+} \equiv\left(B_{r} \backslash B_{\kappa r}\right) \cap\{u>\varepsilon\} .
\end{aligned}
$$

The function $v_{\varepsilon}$ can be obtained by minimizing the Dirichlet integral over $B_{r}$ subject to the above constraints. Also $v_{\varepsilon}$ is continuous at $\{u=\varepsilon\} \cap\left(B_{r} \backslash \bar{B}_{\kappa r}\right)$ and $\min (u, 0)$ $\leqslant v_{\varepsilon} \leqslant u$. Since $\nabla v_{\varepsilon}$ is bounded in $L^{2}\left(B_{r}\right)$, the limit $v=\lim _{\varepsilon \rightarrow 0} v_{\varepsilon}$ exists and $\min (u, 0) \leqslant v \leqslant u$; hence $v$ is continuous in $B_{r}$ and has the desired properties.

We obtain

$$
\begin{aligned}
\int_{B_{r}}\left(|\nabla u|^{2}-|\nabla v|^{2}\right) & \leqslant \int_{B_{r}} q^{2}\left(\lambda^{2}(v)-\lambda^{2}(u)\right) \\
& \leqslant \int_{B_{k r} \cap\{u>0\}} \wedge q^{2}
\end{aligned}
$$


Hence, setting $D^{+}=\left(B_{r} \backslash B_{\kappa r}\right) \cap\{u>0\}$,

$$
\begin{aligned}
\int_{B_{\mathrm{k} r} \cap\{u>0\}}\left(|\nabla u|^{2}-\Lambda q^{2}\right) \leqslant \int_{D^{+}}\left(|\nabla v|^{2}-|\nabla u|^{2}\right) \\
=\int_{D^{+}} \nabla(v-u) \cdot \nabla(u-v+2 v) \\
\leqslant 2 \int_{D^{+}} \nabla v \cdot \nabla(v-u) \\
\leqslant \liminf _{\varepsilon \rightarrow 0} 2 \int_{D^{+}} \nabla v_{\varepsilon} \cdot \nabla\left(v_{\varepsilon}-u\right) \\
=\liminf _{\varepsilon \rightarrow 0} 2 \int_{\partial B_{k r} \cap\{u>\varepsilon\}}(u-\varepsilon)\left|\nabla v_{\varepsilon}\right| \equiv M
\end{aligned}
$$

where in the last formula we have used the integration by parts

$$
\int_{D_{f}^{+}} \nabla v_{\varepsilon} \cdot \nabla\left(v_{\varepsilon}-u\right)=\int_{\partial B_{\kappa r}}(u-\varepsilon)\left|\frac{\partial v_{\varepsilon}}{\partial \nu}\right| ;
$$

notice that $\partial v_{\varepsilon} / \partial \nu \leqslant 0$ on $\partial B_{\kappa r}$. Since $\partial B_{\kappa r}$ and $\partial\{u>\varepsilon\}$ form a corner at their intersection, one has to justify (3.2) by approximation. We shall do this later.

To estimate $M$ we introduce the function $w$ :

$$
\begin{aligned}
\Delta w=0 & \text { in } B_{r} \backslash B_{\kappa r}, \\
w=u & \text { on } \partial B_{r} \cap\{u>\varepsilon\}, \\
w=\varepsilon & \text { elsewhere on } \partial\left(B_{r} \backslash B_{\kappa r}\right) .
\end{aligned}
$$

Clearly $w \geqslant v_{\varepsilon}$ and thus $|\nabla w| \geqslant\left|\nabla v_{\varepsilon}\right|$ on $\partial B_{\kappa r} \cap\{u>\varepsilon\}$. Since

$$
|\nabla w| \leqslant \frac{C}{r} f_{\partial B_{r}}(u-\varepsilon)^{+} \leqslant C \gamma \quad \text { on } \partial B_{\kappa r},
$$

we get

$$
\left|\nabla v_{\varepsilon}\right| \leqslant C \gamma \quad \text { on } \partial B_{\kappa r} \cap\{u>\varepsilon\}
$$

Hence

$$
\begin{aligned}
M \leqslant & C \gamma \int_{\partial B_{\mathrm{k} r}} u^{+} \leqslant C \gamma\left(\int_{B_{\mathrm{k} r}}\left|\nabla u^{+}\right|+\frac{1}{r} \int_{B_{\mathrm{k} r}} u^{+}\right) \\
\leqslant & \frac{C \gamma}{|\Lambda|^{1 / 2} q_{1}} \int_{B_{\mathrm{k} r}}\left(\left|\nabla u^{+}\right|^{2}+|\Lambda| q_{1}^{2} I_{\left.\left\{u^{\prime}, 0\right\}\right\}}\right) \\
& +\frac{C \gamma}{|\Lambda| q_{1}^{2} r}\left(\sup _{B_{\mathrm{k} r}} u^{+}\right) \int_{B_{\mathrm{\kappa} r}}|\Lambda| q_{1}^{2} I_{\left\{u^{+}>0\right\}} .
\end{aligned}
$$


Since $u$ is harmonic in $\{u>0\}, u^{+}$is subharmonic in $\Omega$; therefore $\sup _{B_{k r}} u^{+} \leqslant C \gamma r$. Hence

$$
\begin{aligned}
\int_{\left.B_{\mathrm{k} r} \cap\{u>0)\right\}}\left(|\nabla u|^{2}-\Lambda q_{1}^{2}\right) \leqslant & \frac{C \gamma}{|\Lambda|^{1 / 2} q_{1}}\left(1+\frac{\gamma}{|\Lambda|^{1 / 2} q_{1}}\right) \\
& \cdot \int_{B_{\kappa r} \cap\{u>0\}}\left(|\nabla u|^{2}-\Lambda q_{1}^{2}\right) .
\end{aligned}
$$

Hence if $\gamma /\left(|\Lambda|^{1 / 2} q_{1}\right)$ is small enough then $u \leqslant 0$ in $B_{\kappa r}$.

It remains to justify (3.2). Approximate $D_{\varepsilon}^{+}$by domains $D_{m}$ by changing $D_{\varepsilon}^{+}$near $\partial B_{\kappa r} \cap \partial\{u>\varepsilon\}$ so as to form a smooth boundary there. Denote the corresponding $v_{\varepsilon}$ by $v_{\varepsilon m}\left(v_{\varepsilon m}=\varepsilon\right.$ on the modified boundary $\partial D_{m}$ near $\left.\partial B_{\kappa r} \cap \partial\{u>\varepsilon\}\right)$. Then,

$$
\begin{aligned}
D v_{\varepsilon m} & \rightarrow D v_{\varepsilon} \quad \text { on } \partial B_{\kappa r}, \text { away from } \partial\{u>\varepsilon\}, \\
\left|D v_{\varepsilon m}\right| & \leqslant C \quad \text { on } \partial D_{m}, \text { away from } \partial B_{r}
\end{aligned}
$$

(by (3.3)). Since (3.2) holds for $v_{\varepsilon}=v_{\varepsilon m}$, taking $m \rightarrow \infty$ and using (3.4), the assertion (3.2) for $v_{F}$ follows.

Theorem 3.1 may be considered as a nondegeneracy theorem. It implies

Corollary 3.2. Suppose $\Lambda<0$. If $B_{r} \subset \Omega$ with center in the free boundary $\partial\left\{u>0\right.$; then $\frac{1}{r} f_{\partial B_{r}} u^{+} \geqslant c(c>0) ; c$ depends only on $q_{1}^{2} l_{0}$.

The analog of Theorem 3.1 and its corollary to the case $\Lambda>0$ are obvious.

REMARK 3.1. If $\lambda^{2}(0)<\min \left\{\lambda_{1}^{2}, \lambda_{2}^{2}\right\}$ then the proof of Theorem 3.1 applies to both $u^{+}$and $u^{-}$. Consequently, if $B_{r} \subset \Omega$ with center in the free boundary $\partial\{u>0\}$ $(\partial\{u<0\})$, then

$$
\frac{1}{r} f_{\partial B_{r}} u^{+} \geqslant c \quad\left(\frac{1}{r} f_{\partial B_{r}} u^{-} \geqslant c\right)
$$

where $c$ is a positive constant depending only on $q_{1}^{2}\left\{\min \left(\lambda_{1}^{2}, \lambda_{2}^{2}\right)-\lambda^{2}(0)\right\}$.

4. Upper estimates on the averages. Let

$$
\max \left\{\lambda_{1}^{2}, \lambda_{2}^{2}\right\} \leqslant l_{1} \text {. }
$$

THEOREM 4.1. Assume that $\lambda(0)=\min \left(\lambda_{1}, \lambda_{2}\right)$. There exists a positive constant $C$ depending only on $q_{2}$ (in (3.1)) and $l_{1}$ such that, if $B_{r} \subset \Omega$ with center in $\{u=0\}$, then

$$
\frac{1}{r}\left|f_{\partial B_{r}} u\right| \leqslant C \text {. }
$$

We shall prove the theorem in case $\Lambda<0$; the proof in case $\Lambda>0$ is similar. Since $\Lambda<0, \Delta u$ is a (positive) measure (by Theorem 2.3). In order to prove the theorem we first estimate the measure $\Delta u$.

Lemma 4.2. If $\Lambda<0$ and $B_{r} \subset \Omega$ then

$$
\Delta u\left(B_{r / 2}\right) \leqslant C r^{n-1} .
$$


Proof. Defining $v$ as in (2.1) we have

$$
\int_{B_{r}}|\nabla u|^{2}-\int_{B_{r}}|\nabla v|^{2} \leqslant \int_{B_{r}}\left(Q^{2}(v, x)-Q^{2}(u, x)\right) \leqslant C r^{n} .
$$

The left-hand side is equal to

$$
\int_{B_{r}} \nabla(u-v) \cdot \nabla(u+v)=\int_{B_{r}} \nabla(u-v) \cdot \nabla u=\int_{B_{r}}(v-u) \Delta u
$$

where $\Delta u$ is a measure supported on $\{u=0\}$ (the continuity of $v-u$ is used in making sense out of the last integral); the last integral is equal to $\int_{B_{r}} v \Delta u$. Since $v \geqslant u$, also $v \Delta u \geqslant u \Delta u=0$, and thus

$$
\int_{B_{r / 2} \cap\{u=0\}} v \Delta u \leqslant C r^{n}
$$

We shall use the representation

$$
u\left(x^{0}\right)=\int_{\partial B_{r}} P_{x^{0}}(y) u(y)-\int_{B_{r}} G_{x^{0}}(y) \Delta u(y)
$$

where $P_{x^{0}}$ and $G_{x^{0}}$ are Poisson's kernel and Green's function (in $B_{r}$ ), respectively. This formula can be justified by approximating $u$ by mollifiers $J_{\varepsilon} u$, applying the formula to $J_{\varepsilon} u$ at $x^{0}$ and taking $\varepsilon \rightarrow 0$. If $x^{0} \in\{u=0\}$ then we obtain, from (4.5),

$$
\int_{B_{r}} G_{x^{0}}(y) \Delta u(y)=\int_{\partial B_{r}} P_{x^{0}}(y) u(y)
$$

and the right-hand side is precisely $v\left(x^{0}\right)$. Thus we can rewrite (4.4) in the form

$$
\int_{B_{r / 2}}\left(\int_{B_{r}} G_{x}(y) \Delta u(y)\right) \Delta u(x) \leqslant C r^{n} .
$$

Noting that $G_{x}(y) \geqslant c r^{2-n}$ if $x, y \in B_{r / 2}(c>0)$ we obtain $c r^{2-n}\left(\Delta u\left(B_{r / 2}\right)\right)^{2} \leqslant C r^{n}$, and the assertion (4.3) follows.

Proof of Theorem 4.1. As before we take $\Lambda<0$. We may assume that the center of $B_{r}$ is in the origin. By (4.6),

$$
\int_{\partial B_{r}} P_{0} u=\int_{B_{r}} G_{0}(y) \Delta u(y) .
$$

Suppose first that $\Delta u$ is smooth. Then

$$
I \equiv \int_{B_{r}} G_{0}(y) \Delta u(y)=\int_{0}^{r} G(s) h(s) d s
$$

with suitable functions $G$ and $h ; h(r)=r^{n-1} \int_{\partial B_{1}} \Delta u(r \xi) d H^{n-1}(\xi)$. By Lemma 4.2,

$$
\int_{0}^{s} h(\tau) d \tau \leqslant C s^{n-1}
$$

Hence,

$$
I=\int_{0}^{r} G(s) \frac{d}{d s}\left(\int_{0}^{s} h(\tau) d \tau\right) d s=\left[G(s) \int_{0}^{s} h(\tau) d \tau\right]_{0}^{r}-\int_{0}^{r} G^{\prime}(s) \int_{0}^{s} h(\tau) d \tau d s .
$$


The expression in brackets vanishes at $s=r$ (since $G_{0}=0$ on $\partial B_{r}$ ) and at $s=0$ (by (4.8) and $\left.G(s) \leqslant C s^{2-n}\right)$. Hence,

$$
\int_{B_{r}} G_{0}(y) \Delta u(y) \leqslant \int_{0}^{r} \frac{C}{s^{n-1}} C s^{n-1} d s \leqslant C r .
$$

By using mollifiers $u_{\varepsilon}=u * \psi_{\varepsilon}$ we can establish the same estimate for the measure $\Delta u$. Here we use the estimate

$$
\begin{aligned}
& \int_{B_{r}\left(x_{0}\right)} \Delta u_{\varepsilon}(x)=\int_{B_{r}\left(x_{0}\right)} \int_{\{|\cdot|<\varepsilon\}} \Delta u(x-y) \psi_{\varepsilon}(y) d y \\
& =\int_{\{|\eta|<\varepsilon\}} \int_{B_{r}\left(x_{0}\right)} \Delta u(x-y) \psi_{\varepsilon}(y) d y \\
& =\int_{\{||||<\varepsilon\}} \Delta u\left(B_{r}\left(x_{0}-y\right)\right) \psi_{\varepsilon}(y) d y \leqslant C r^{n-1} .
\end{aligned}
$$

From (4.7) and (4.9) we see that $\frac{1}{r} f_{\partial B} u \leqslant C$. Since $u(0)=0$ and $u$ is subharmonic, the last integral is actually positive and therefore (4.2) follows.

\section{Lipschitz continuity.}

Lemma 5.1. Let $u$ be any function in $C^{0}\left(B_{R}\right) \cap H^{1.2}\left(B_{R}\right)$, where $B_{r}$ is a ball with radius $r$ and center $x^{0}, u\left(x^{0}\right)=0$, and $u$ is harmonic in $B_{R} \backslash\{u=0\}$. Set

$$
\phi(r)=\frac{1}{r^{2}} \int_{B_{r}} \rho^{2-n}\left|\nabla u^{+}\right|^{2} d x \cdot \frac{1}{r^{2}} \int_{B_{r}} \rho^{2-n}\left|\nabla u^{-}\right|^{2} d x
$$

where $\rho=\left|x-x^{0}\right|$. Then $\phi(r)<\infty$ and $\phi(r)$ is increasing in $r, r \in(0, R)$.

We shall refer to this result as the monotonicity lemma.

Proof. Set $S_{r}=\partial B_{r}$. We first assume that

$$
\min _{S_{r}} u<0<\max _{S_{r}} u \text { for all } r \in(0, R) .
$$

Notice that the distribution $\Delta u^{+}$is a measure. Denote by $v_{m}$ mollifiers of $u^{+}$. Then $\Delta v_{m}^{2}=2\left|\nabla v_{m}\right|^{2}+2 v_{m} \Delta v_{m} \geqslant 2\left|\nabla v_{m}\right|^{2}$, so that

$$
2 \int_{B_{r} \backslash B_{r}}\left|\nabla v_{m}\right|^{2} \rho^{2-n} \leqslant \int_{B_{r} \backslash B_{r}} \Delta\left(v_{m}^{2}\right) \rho^{2-n}=r^{2-n} \int_{S_{r}} \frac{\partial}{\partial r} v_{m}^{2}+(n-2) r^{1-n} \int_{S_{r}} v_{m}^{2}-I_{F}
$$

where

$$
I_{\varepsilon}=\varepsilon^{2-n} \int_{S_{\xi}} \frac{\partial}{\partial r} v_{m}^{2}+(n-2) \varepsilon^{1-n} \int_{S_{\varepsilon}} v_{m}^{2}
$$

Since $\left|D v_{m}\right|$ is bounded, $I_{\varepsilon} \rightarrow(n-2)\left|S_{1}\right| v_{m}^{2}(0)$ as $\varepsilon \rightarrow 0$. Hence,

$$
2 \int_{B_{r} \backslash B_{\varepsilon}}\left|\nabla v_{m}\right|^{2} \rho^{2-n} \leqslant r^{2-n} \int_{S_{r}} \frac{\partial}{\partial r} v_{m}^{2}+(n-2) r^{1-n} \int_{S_{r}} v_{m}^{2} .
$$


Integrating with respect to $r, r_{0}<r<r_{0}+\delta$, and dividing by $\delta$, and then letting $m \rightarrow \infty$, we obtain

$$
\begin{aligned}
\frac{2}{\delta} \int_{r_{0}}^{r_{0}+\delta} d r \int_{B_{r} \backslash B_{\varepsilon}}\left|\nabla u^{+}\right|^{2} \rho^{2-n} \leqslant & \frac{1}{\delta} \int_{r_{0}}^{r_{0}+\delta} r^{2-n} d r \int_{S_{r}} 2 u^{+} u_{r}^{+} \\
& +\frac{n-2}{\delta} \int_{r_{0}}^{r_{0}+\delta} r^{1-n} d r \int_{S_{r}}\left(u^{+}\right)^{2}
\end{aligned}
$$

Taking $\delta \rightarrow 0$ we obtain for a.a. $r_{0}$

$$
2 \int_{B_{r_{0}} \backslash B_{r}} \mid \nabla u^{+} P^{2} \rho^{2-n} \leqslant r_{0}^{2-n} \int_{S_{r_{0}}} 2 u^{+} u_{r}^{+}+(n-2) r_{0}^{1-n} \int_{S_{r_{0}}}\left(u^{+}\right)^{2}
$$

Hence, for a.a. $r$,

$$
2 \int_{B_{r}}\left|\nabla u^{+}\right|^{2} \rho^{2-n} \leqslant r^{2-n} \int_{S_{r}} 2 u^{+} u_{r}^{+}+(n-2) r^{1-n} \int_{S_{r}}\left(u^{+}\right)^{2}
$$

Since a similar inequality holds for $u^{-}$, it follows that $\psi(r)$ is finite.

Since $r \rightarrow \int_{S_{r}}\left|\nabla u^{+}\right|^{2} \rho^{2-n}$ is in $L^{1}(0, R)$, we have

$$
\frac{d}{d r} \int_{B_{r}} \rho^{2-n}\left|\nabla u^{+}\right|^{2}=\int_{S_{r}} r^{2-n}\left|\nabla u^{+}\right|^{2} \text { a.e. }
$$

It follows that a.e.

$$
\begin{aligned}
\phi^{\prime}(r)= & -\frac{4}{r^{5}} \int_{B_{r}} \rho^{2-n}\left|\nabla u^{+}\right|^{2} \cdot \int_{B_{r}} \rho^{2-n}\left|\nabla u^{-}\right|^{2}+\frac{1}{r^{4}} \int_{S_{r}} r^{2-n}\left|\nabla u^{+}\right|^{2} \\
& \cdot \int_{B_{r}} \rho^{2-n}\left|\nabla u^{-}\right|^{2}+\frac{1}{r^{4}} \int_{B_{r}} \rho^{2-n}\left|\nabla u^{+}\right|^{2} \cdot \int_{S_{r}} r^{2-n}\left|\nabla u^{-}\right|^{2}
\end{aligned}
$$

We shall prove that $\phi^{\prime}(r) \geqslant 0$ a.e. in $(0, R)$. By scaling, we may assume that $r=1$.

Denote by $\nabla_{\theta} v$ the gradient of a function $v$ on $S_{1}$. Denote by $\Gamma_{1}$ the support of $u^{+}$ on $S_{1}$, and by $\Gamma_{2}$ the support of $u^{-}$on $S_{1}$. By assumption,

$$
\operatorname{meas}\left(\Gamma_{i}\right) \neq 0 \text { for } i=1,2, \ldots
$$

We introduce the constants

$$
\frac{1}{\alpha_{i}}=\inf _{v \in H_{0}^{1.2}\left(\Gamma_{1}\right)} \frac{\int_{\Gamma_{1}}\left|\nabla_{\theta} v\right|^{2}}{\int_{\Gamma_{1}} v^{2}}
$$

For any $0<\beta_{1}<1$ we can write

$$
\begin{aligned}
\int_{S_{1}}\left(\left(u_{r}^{+}\right)^{2}+\beta_{1}^{2}\left|\nabla_{\theta} u^{+}\right|^{2}\right) & \geqslant 2\left\{\int_{S_{1}}\left(u_{r}^{+}\right)^{2} \cdot \int_{S_{1}} \beta_{1}^{2}\left|\nabla_{\theta} u^{+}\right|^{2}\right\}^{1 / 2} \\
& \geqslant 2 \frac{\beta_{1}}{\sqrt{\alpha_{1}}}\left\{\int_{S_{1}}\left(u_{r}^{+}\right)^{2} \cdot \int_{S_{1}}\left(u^{+}\right)^{2}\right\}^{1 / 2} \geqslant \frac{2 \beta_{1}}{\sqrt{\alpha_{1}}} \int_{S_{1}}\left|u^{+} u_{r}^{+}\right|
\end{aligned}
$$


and

$$
\int_{S_{1}}\left(1-\beta_{1}^{2}\right)\left|\nabla_{\theta} u^{+}\right|^{2} \geqslant \frac{1-\beta_{1}^{2}}{\alpha_{1}} \int_{S_{1}}\left(u^{+}\right)^{2}
$$

Choosing

$$
\frac{1-\beta_{i}^{2}}{\alpha_{i}}=(n-2) \frac{\beta_{i}}{\sqrt{\alpha_{i}}}
$$

we find that

$$
\int_{S_{1}}\left|\nabla u^{+}\right|^{2} \geqslant \frac{\beta_{1}}{\sqrt{\alpha_{1}}}\left\{\int_{S_{1}} 2\left|u^{+} u_{r}^{+}\right|+(n-2) \int_{S_{1}}\left(u^{+}\right)^{2}\right\} .
$$

The relations (5.2) and (5.6) hold also for $u^{-}$. Comparing with (5.3) we see that $\phi^{\prime}(r) \geqslant 0$ provided

$$
\frac{\beta_{1}}{\sqrt{\alpha_{1}}}+\frac{\beta_{2}}{\sqrt{\alpha_{2}}} \geqslant 2
$$

We easily compute that the $\beta_{i}$ satisfy (5.5) if

$$
\frac{\beta_{i}}{\sqrt{\alpha_{i}}}=\frac{1}{2}\left\{\left[(n-2)^{2}+\frac{4}{\alpha_{i}}\right]^{1 / 2}-(n-2)\right\} .
$$

If $\gamma_{i}$ is defined by

$$
\gamma_{i}\left(\gamma_{i}+n-2\right)=1 / \alpha_{i}, \quad \gamma_{i}>0
$$

then we obtain

$$
\frac{\beta_{i}}{\sqrt{\alpha_{i}}}=\gamma_{i}
$$

The set function $\gamma_{1}$ as a function of $\Gamma_{1}$ was studied by Sperner [12] and by Friedland and Hayman [8]. In [12] it is proved that $\gamma_{1}(E) \geqslant \gamma_{1}\left(E^{*}\right)$ where $E$, $E^{*} \subset S_{1}$ provided $E^{*}$ is a spherical cap having the same $(n-1)$-dimensional Hausdorff measure as $E$. In [8] it is proved that $\gamma_{1}(E) \geqslant \psi(s)$ where $s=$ meas $(E) / \operatorname{meas}\left(S_{1}\right)$, and $\psi(s)$ is convex and decreasing:

$$
\psi(s)= \begin{cases}\frac{1}{2} \log \frac{1}{4 s}+\frac{3}{2} & \text { if } s<\frac{1}{4} \\ 2(1-s) & \text { if } \frac{1}{4}<s<1 .\end{cases}
$$

Setting $s_{1}=\operatorname{meas}\left(\Gamma_{i}\right) / \operatorname{meas}\left(S_{1}\right)$, we then have

$$
\gamma_{1}+\gamma_{2} \geqslant \psi\left(s_{1}\right)+\psi\left(s_{2}\right) \geqslant 2 \psi\left[\left(s_{1}+s_{2}\right) / 2\right] \geqslant 2 \psi(1 / 2)=2
$$

in view of (5.9), this completes the proof of (5.7), provided (5.1) is satisfied.

If (5.1) is not satisfied, let $R_{0}$ be the smallest value of $r$ for which at least one of the inequalities in (5.1) is invalid. Suppose for definiteness that $\min _{S_{R_{0}}} u \geqslant 0$. Then 
$u^{-}$is harmonic in $D=B_{R_{0}} \cap\{u<0\}$, vanishing on $\partial D$; hence $u^{-}=0$ in $D$, which gives $\phi(r)=0$ if $0<r \leqslant R_{0}$. Since $\phi^{\prime}(r) \geqslant 0$ for a.a. $R_{0}<r<R$ (by the previous proof), the proof of the lemma is complete.

We shall now use Theorem 4.1 in order to establish Lipschitz continuity for any minimizer $u$.

Lemma 5.2. Assume that $\lambda(0)=\min \left(\lambda_{1}, \lambda_{2}\right)$. Then for any domain $D \Subset \Omega$ there exists a positive constant $C$ such that if $B_{r} \subset D$ with center in $\{u=0\}$ then

$$
\frac{1}{r} f_{\partial B_{r}}|u| \leqslant C \text {. }
$$

Proof. By Green's formula $(0<\alpha<1)$

$$
\begin{aligned}
f_{\partial B_{r}} r^{\alpha} u^{-} & =\int_{B_{r}} G_{0} \Delta\left(\rho^{\alpha} u^{-}\right)=-\int_{B_{r}} \nabla G_{0} \cdot \nabla\left(\rho^{\alpha} u^{-}\right) \\
& =-\int_{B_{r}} \rho^{\alpha} \nabla G_{0} \cdot \nabla u^{-}+c_{n} \int_{B_{r}} \rho^{1-n} \alpha \rho^{\alpha-1} u^{-} \equiv J_{1}+J_{2},
\end{aligned}
$$

and $G_{0}(\rho)=c \rho^{2-n}, c>0$ (we take for definiteness $n \geqslant 3$ ). Clearly,

$$
\begin{aligned}
\left|J_{1}\right| & \leqslant C\left(\int_{B_{r}} \rho^{2-n}\left|\nabla u^{-}\right|^{2}\right)^{1 / 2}\left(\int_{B_{r}} \rho^{2 \alpha-n}\right)^{1 / 2} \\
& \leqslant C r^{\alpha}\left(\int_{B_{r}} \rho^{2-n}\left|\nabla u^{-}\right|^{2}\right)^{1 / 2} .
\end{aligned}
$$

Introducing the function $\phi_{\varepsilon}(r)=\left(r^{\varepsilon} / r\right) \int_{\partial B_{r}} u^{-}(0<\varepsilon<\alpha)$ we also have

$$
J_{2} \leqslant c_{n} \alpha \int_{0}^{r} \rho^{\alpha-\varepsilon} \phi_{\varepsilon}(\rho) \leqslant \frac{c_{n} \alpha}{1+\alpha-\varepsilon} r^{1+\alpha-\varepsilon} \sup _{\rho \leqslant r} \phi_{\varepsilon}(\rho) ;
$$

notice that $\phi_{\varepsilon}(\rho)$ is bounded since $u^{-}$is Hölder continuous with any exponent $<1$. Dividing both sides of (5.11) by $r^{1+\alpha-\varepsilon}$ we then have

$$
\phi_{\varepsilon}(r) \leqslant c_{n} \alpha \sup _{\rho \leqslant r} \phi_{\varepsilon}(\rho)+\frac{c r^{\varepsilon}}{r}\left(\int_{B_{r}} \rho^{2-n}\left|\nabla \cdot u^{-}\right|^{2}\right)^{1 / 2} .
$$

Similarly, if $\left.\psi_{\varepsilon}(r)=\left(r^{\varepsilon} / r\right)\right\}_{\partial B_{r}} u^{+}$then

$$
\psi_{\varepsilon}(r) \leqslant c_{n} \alpha \sup _{\rho \leqslant r} \psi_{\varepsilon}(\rho)+\frac{C r^{\varepsilon}}{r}\left(\int_{B_{r}} \rho^{2-n}\left|\nabla u^{+}\right|^{2}\right)^{1 / 2} .
$$

By Theorem $4.1 \phi_{\varepsilon}(r)=\psi_{\varepsilon}(r)+O\left(r^{\varepsilon}\right)$. Hence,

$$
\phi_{\varepsilon}(r) \leqslant c_{n} \alpha \sup _{\rho \leqslant r} \phi_{\varepsilon}(\rho)+\frac{C r^{\varepsilon}}{r}\left(\int_{B_{r}} \rho^{2-n}\left|\nabla u^{+}\right|^{2}\right)^{1 / 2}+O\left(r^{\varepsilon}\right) .
$$

Taking the product of the left-hand sides of (5.12) and (5.13), we obtain

$$
\left(\phi_{\varepsilon}(r)\right)^{2} \leqslant C \alpha^{2}\left(\sup _{\rho \leqslant r} \phi_{\varepsilon}(\rho)\right)^{2}+C r^{2 \varepsilon}+C \frac{r^{2 \varepsilon}}{r^{2}}\left(\int_{B_{r}} \rho^{2-n}\left|\nabla u^{+}\right|^{2} \cdot \int_{B_{r}} \rho^{2-n}\left|\nabla u^{-}\right|^{2}\right)^{1 / 2} .
$$


Using Lemma 5.1 and choosing $\alpha$ small enough, we obtain $\left(\phi_{\varepsilon}(r)\right)^{2} \leqslant C r^{2 \varepsilon}, C$ independent of $\varepsilon$. Hence, $\frac{1}{r} f_{\partial B_{r}} u^{-} \leqslant C$ and therefore, also, $\frac{1}{r} f_{\partial B_{r}} u^{+} \leqslant C$.

THEOREM 5.3. If $\lambda(0)=\min \left(\lambda_{1}, \lambda_{2}\right)$, then $u$ is Lipschitz continuous in any compact subset of $\Omega$.

Proof. Let $K$ be a compact subset of $\Omega$ and introduce $d=\operatorname{dist}(K, \partial \Omega)$. For any $x \in \Omega, x \notin\{u=0\}$, denote by $\rho=\rho(x)$ the distance from $x$ to $\{u=0\}$ and let $x^{0}$ be such that $\rho=\left|x-x^{0}\right|, u\left(x^{0}\right)=0$. If $\rho>d / 6$ then $u$ is harmonic in $B_{d / 6}(x)$, and thus $|D u(x)| \leqslant C / d$. Suppose next that $\rho(x)<d / 6$.

Representing $u$ by Poisson's formula, $u(x)=\int_{\partial B_{\rho}(x)} P_{x}(y) u(y)$, we conclude that

$$
|D u(x)| \leqslant \frac{C}{\rho} f_{\partial B_{\rho}(x)}|u(y)| .
$$

The function $|u|$ is subharmonic. Representing $|u(y)|\left(y \in \partial B_{\rho}(x)\right)$ by Green's function in $B_{\sigma}\left(x^{0}\right)$ we get

$$
|u(y)| \leqslant \oint_{\partial B_{\sigma}\left(x^{0}\right)} \tilde{P}_{y}(z)|u(z)|, \quad 3 \rho<\sigma<5 \rho ;
$$

thus, $|u(y)| \leqslant C f_{\partial B_{o}\left(x^{0}\right)}|u(z)| \leqslant C \rho$ by Lemma 5.2. Substituting this into (5.14) we conclude that $|D u(x)| \leqslant C$ if $x \in K, u(x) \neq 0$. Since $u \in H_{\text {loc }}^{1,2}(\Omega), D u=0$ a.e. on $\{u=0\}$, and thus $D u \in L^{\infty}(K)$.

Another Proof of Theorem 5.3. We shall give another proof, also based on Theorem 4.1 and Lemma 5.1.

Suppose $0 \in \Omega, u(0)=M>0$, and let $x^{0}$ be the nearest point to 0 on $\{u=0\}$. We assume first that $\left|x^{0}\right|=1$ and $B_{2} \subset \Omega$. By Harnack's inequality $u>c_{0} M$ in $B_{3 / 4}$ $\left(c_{0}>0\right)$ and therefore $f_{\partial B_{1}\left(x^{0}\right)} u^{+}>c M(c>0)$. From Theorem 4.1 it follows that

$$
\int_{\partial B_{1}\left(x^{0}\right)} u^{-}>c M
$$

with another $c>0$, provided $M$ is large enough.

Let $y \in \partial B_{1 / 2}$ be a point on $\overrightarrow{0 x^{0}}$. Then $u>c_{0} M>0$ in $B_{1 / 4}(y)$. We shall use polar coordinates $(r, \omega)$ about $y$. Denote by $\Gamma$ the set of $\omega$ 's such that if $(r, \omega) \in$ $\partial B_{1}\left(x^{0}\right)$ then $u(r, \omega)<0$.

We integrate $u_{r}^{-}(r, \omega)$ over $(r, \omega) \in B_{1}\left(x^{0}\right), \omega \in \Gamma$. Using (5.15) and the fact that $u>0$ in $B_{1 / 4}(y)$ we obtain

$$
c M \leqslant \int_{\partial B_{1}(y)} u^{-}=\int_{\Gamma} d \omega \int u_{r}^{-} \leqslant|\Gamma|^{1 / 2}\left\{\int_{B_{1}\left(x^{0}\right)}\left|\nabla u^{-}\right|^{2}\right\}^{1 / 2} .
$$

Next we integrate $u_{r}^{+}(r, \omega)$ and $(r, \omega) \in\left\{B_{1}\left(x^{0}\right) \backslash B_{1 / 4}(y)\right\}, \omega \in \Gamma$, and notice that $u^{+}(r, \omega) \geqslant c_{0} M$ in $B_{1 / 4}(y)$. We obtain

$$
c M|\Gamma| \leqslant \int_{\Gamma} d \omega \int u_{r}^{+} \leqslant|\Gamma|^{1 / 2}\left\{\int_{B_{1}\left(x^{0}\right)}\left|\nabla u^{+}\right|^{2}\right\}^{1 / 2} .
$$


Taking the product of both sides of the inequalities in (5.16) and (5.17), we get

$$
c M^{4} \leqslant \int_{B_{1}\left(x^{0}\right)}\left|\nabla u^{+}\right|^{2} \cdot \int_{B_{1}\left(x^{0}\right)}\left|\nabla u^{-}\right|^{2} .
$$

Using Lemma 5.1 we then obtain $M \leqslant C$. We have thus proved that

$$
u(z) \leqslant C \rho(z) \quad(\rho(z)=\operatorname{dist}(z,\{u=0\}))
$$

if $z=0, \rho(z)=1, u(z)>0, B_{2 \rho(z)}(z) \subset \Omega$. The proof for general $z$ follows by considering $\tilde{u}(x) \equiv u(z+\rho(z) x) / \rho(z)$. From (5.18) we deduce that $|\nabla u(z)| \leqslant C$; the same estimate holds if $u(z)<0$. The proof that $u \in C_{\mathrm{l} \alpha c}^{0.1}$ now readily follows.

6. Blow-up limits. The rest of this paper is devoted to the study of the free boundary. For definiteness we shall always assume that

$$
\Lambda<0, \quad \lambda(0)=\lambda_{1} ;
$$

all the results obviously extend to the case $\Lambda>0, \lambda(0)=\lambda_{2}$. When (6.1) holds the free boundary coincides with

$$
\Gamma^{+}=\partial\{u>0\}
$$

Indeed, for the remaining free boundary

$$
\Gamma^{-}=\partial\{u<0\} \backslash \partial\{u>0\},
$$

we obviously have

$$
u \leqslant 0 \text { in a neighborhood } N \text { of } \Gamma^{-} .
$$

But since $\lambda(0)=\lambda_{1}$, the minimizer $u$ must be harmonic in $N$. Consequently, $\Gamma$ is empty.

Definition 6.1. A function $u$ is called a minimizer (of $J$ ) in $R^{\prime \prime}$ if for any $B_{r} \subset R^{\prime \prime}$ and for any $v \in H^{1.2}\left(B_{r}\right), v=u$ on $\partial B_{r}$.

$$
J_{B_{r}}(u) \leqslant J_{B_{r}}(v)
$$

where $J_{B_{r}}(v)$ is the functional $J(v)$ with $\Omega$ replaced by $B_{r}$.

Suppose $u$ is a minimizer, $u\left(x_{k}\right)=0, x_{k} \rightarrow x_{0} \in \Omega, \rho_{k} \downarrow 0$, and set

$$
u_{k}(x)=\frac{1}{\rho_{k}} u\left(x_{i}+\rho_{k} x\right) \text {. }
$$

We call $\left\{u_{k}\right\}$ a blow-up sequence with respect to $B_{\rho_{k}}\left(x_{k}\right)$. Since $\left|\nabla u_{k}(x)\right| \leqslant C$ in any bounded set and $u_{k}(0)=0$, we have, for a subsequence,

$$
\begin{aligned}
u_{k}(x) & \rightarrow u_{0}(x) \quad \text { unformly in bounded sets, } \\
\nabla u_{k} & \rightarrow \nabla u_{0} \quad \text { weakly in } L_{\mathrm{loc}}^{\infty}\left(R^{n}\right) ;
\end{aligned}
$$

$u_{0}$ is called a blow-up limit.

LEMMA 6.1. There holds

$$
\begin{gathered}
\partial\left\{u_{k}>0\right\} \rightarrow \partial\left\{u_{0}>0\right\} \quad \text { locally in the Hausdorff metric, } \\
\nabla u_{k} \rightarrow \nabla u_{0} \quad \text { a.e. in } R^{n}
\end{gathered}
$$


Proof. Suppose a ball $\bar{B}_{r}$ does not intersect $\partial\left\{u_{0}>0\right\}$. Then either $u_{0}>0$ in $\bar{B}_{r}$ or $u_{0} \leqslant 0$ in $\bar{B}_{r}$. In the first case $u_{k}>0$ in $\bar{B}_{r}$ if $k$ is large enough. In the second case $\frac{1}{r} f_{\partial B_{r}} u_{k}^{+}<\varepsilon$ for any $\varepsilon>0$ if $k$ is large enough, so that, by nondegeneracy, $u_{k} \leqslant 0$ in $B_{r / 2}$.

In both cases we conclude that $B_{r / 2}$ does not intersect $\partial\left\{u_{k}>0\right\}$ if $k$ is large enough.

Conversely, if $B_{r}$ does not intersect $\partial\left\{u_{k}>0\right\}$ for any large $k$ then either $u_{k}>0$ in $B_{r}$ or $u_{k} \leqslant 0$ in $B_{r}$. In the first case $u_{k}$ is harmonic in $B_{r}$ and then so is $u_{0}$; thus either $u_{0}>0$ in $B_{r}$ or $u_{0} \equiv 0$ in $B_{r}$, so that $B_{r}$ does not intersect $\partial\left\{u_{0}>0\right\}$. In the second case we have $u_{0} \leqslant 0$ in $B_{r}$ so that again $B_{r}$ does not intersect $\partial\left\{u_{0}>0\right\}$.

To prove (6.8) notice that, in every compact subset of $\left\{u_{0} \neq 0\right\},(6.8)$ is certainly valid. Next consider a density point $x^{0}$ of the set $\left\{u_{0}(x)=0\right\}$. By the Lipschitz continuity of $u_{0}$, we then deduce that $\left|u_{0}\right|=o(r)$ in $B_{r}$, and therefore, $\frac{1}{r} \int_{\partial B_{r}} u_{0}^{+}=o(1)$ as $r \rightarrow 0$.

Since $u_{k} \rightarrow u_{0}$ uniformly in $B_{1}$, we get $\frac{1}{r} \int_{\partial B_{r}} u_{k}^{+}<\varepsilon$ for any small $\varepsilon>0$, provided $k$ is large enough; hence by nondegeneracy, $u_{k} \leqslant 0$ in $B_{r}$. But then (since $\left.\lambda(0)=\lambda_{1}\right) u_{k}$ is harmonic in $B_{r}$ and then so is $u_{0}$. Consequently, $\nabla u_{k} \rightarrow \nabla u_{0}$ uniformly in $B_{r / 2}$. We have thus proved that almost all the set $\left\{u_{0}=0\right\}$ can be covered by balls $B_{r}$, with suitable centers such that $\nabla u_{k} \rightarrow \nabla u_{0}$ in each $B_{r_{i}}$. It follows that $\nabla u_{k} \rightarrow \nabla u_{0}$ a.e. in the set $\left\{u_{0}=0\right\}$. This completes the proof of (6.8).

LemMA 6.2. $u_{0}$ is a minimizer in $R^{n}$ with respect to the function $Q_{0}(u, \lambda)=$ $q\left(x_{0}\right) \lambda(u)$.

Indeed, the proof is similar to the proof of Lemma 5.4 in [1]; that proof can be slightly simplified by using (6.8).

Theorem 6.3. Suppose $D \Subset \Omega, B_{r} \subset D$ with center $x^{0}$ in $\partial\{u>0\}$. Then

$$
\frac{1}{r} f_{\partial B_{r}\left(x^{0}\right)} u \geqslant c, \quad c>0 \text {. }
$$

Strictly speaking, this result does not include Corollary 3.2 since the constant $c$ in (6.9) depends also on $D$ and on the Lipschitz coefficient of $u$.

Proof. Suppose the assertion is not true. Then there exist points $x_{m}^{0} \in D$ and $r_{m} \downarrow 0$ such that

$$
\frac{1}{r_{m}} f_{\partial B_{r_{m}}\left(x_{m}^{0}\right)} u \rightarrow 0, \quad x_{m}^{0} \in \partial\{u>0\}
$$

Setting $u_{m}(x)=u\left(x_{m}^{0}+r_{m} x\right) / r_{m}$ we may suppose that $x_{m}^{0} \rightarrow 0, u_{m} \rightarrow u_{0}$ uniformly in bounded sets. Then $u_{0}$ is subharmonic (since $u_{m}$ is subharmonic) and $f_{\partial B_{1}} u_{0}=0$ $=u_{0}(0)$. By the maximum principle it then follows that $u_{0}$ is harmonic in $B_{1}$.

Now $u_{0}$ is a local minimizer and $0 \in \partial\left\{u_{0}>0\right\}$, by (6.7). It follows that the free boundary $\partial\left\{u_{0}>0\right\}$ is nonempty; this set must be piecewise analytic since $u_{0}$ is harmonic. But then Theorem 2.4 shows that $\left|\nabla u_{0}\right|^{2}$ has jump $\Lambda q^{1}(0)$ across smooth parts of the free boundary. Since, however, $u_{0}$ is harmonic, $\left|\nabla u_{0}\right|^{2}$ cannot have a jump, i.e., $\Lambda q^{2}(0)=0$, a contradiction. 
Consider a blow-up family

$$
u_{\varepsilon}(x)=\frac{1}{\varepsilon} u\left(x^{0}+\varepsilon x\right), \quad x^{0} \in \partial\{u>0\}, \varepsilon>0
$$

and let

$$
\begin{aligned}
I_{\varepsilon}(r) & \equiv \frac{1}{r^{4}} \int_{B_{r}} \rho^{2-n}\left|\nabla u_{\varepsilon}^{+}\right|^{2} \cdot \int_{B_{r}} \rho^{2-n}\left|\nabla u_{\varepsilon}^{-}\right|^{2} \\
& =\frac{1}{(\varepsilon r)^{4}} \int_{B_{\varepsilon r}\left(x^{0}\right)} \rho^{2-n}\left|\nabla u^{+}\right|^{2} \cdot \int_{B_{\varepsilon r}\left(x^{0}\right)} \rho^{2-n}\left|\nabla u^{-}\right|^{2} \equiv \tilde{I}_{\varepsilon r}
\end{aligned}
$$

By Lemma 5.1, $\tilde{I}_{\rho}$ is an increasing function of $\rho$. Consequently there exists a nonnegative constant $\gamma$ such that

$$
I_{\varepsilon}(r) \downarrow \gamma \quad \text { if } \varepsilon \downarrow 0 .
$$

Now take a sequence $\varepsilon=\varepsilon_{k} \downarrow 0$ such that

$$
u_{\varepsilon_{h}}(x) \rightarrow u_{0}(x) \text { uniformly in bounded subsets of } R^{n} \text {. }
$$

LEMMA 6.4. If (6.11) holds then, as $\varepsilon_{k} \downarrow 0$,

$$
I_{\varepsilon_{h}}(r) \rightarrow \frac{1}{r^{4}} \int_{B_{r}} \rho^{2-n}\left|\nabla u_{0}^{+}\right|^{2} \cdot \int_{B_{r}} \rho^{2-n}\left|\nabla u_{0}^{-}\right|^{2} .
$$

Proof. By the Lipschitz continuity of $u,\left|\nabla u_{\varepsilon}^{+}\right| \leqslant C$, and by Lemma 6.1, $\nabla u_{\varepsilon}^{ \pm} \rightarrow$ $\nabla u_{0}^{ \pm}$a.e. Hence, (6.12) follows by the Lebesgue bounded convergence theorem.

COROLlaRY 6.5. For any blow-up limit $u_{0}$ of $u_{\varepsilon}$ there holds

$$
\frac{1}{r^{4}} \int_{B_{r}} \rho^{2-n}\left|\nabla u_{0}^{+}\right|^{2} \cdot \int_{B_{r}} \rho^{2-n}\left|\nabla u_{0}^{-}\right|^{2}=\gamma
$$

for all $r>0$.

LEMMA 6.6. (i) If $\gamma=0$ then $u_{0} \geqslant 0$ in $R^{n}$; (ii) if $\gamma>0$ and $n=2$ then $u_{0}(x)=$ $\mu_{2}(x \cdot e)^{+}-\mu_{1}(x \cdot e)^{-}$in $R^{n}$ where $e$ is a constant unit vector, $\mu_{i}$ are positive constants, and $\mu_{1}^{2}-\mu_{2}^{2}=\Lambda q^{2}\left(x^{0}\right)$.

The function $u_{0}$ in (ii) is called a 2-plane solution; if $\mu_{1}=0$ or $\mu_{2}=0$ then we call it a 1-plane solution.

Proof. If $\gamma=0$ then either $u_{0}^{+}=0$ or $u_{0}^{-}=0$ in $R^{n}$. Since $u_{0}$ is subharmonic and $u_{0}(0)=0$, we conclude that $u_{0} \geqslant 0$. To prove (ii) we check the proof of Lemma 5.1 and find that equality can hold in (6.13) only if equality holds in the various Cauchy-Schwarz inequalities and $s_{1}=s_{2}=1 / 2$. Thus, with $S_{1}$ replaced by $S_{r}$,

$$
\begin{gathered}
\left|u_{r}^{+}\right|=C u^{+}, \quad u^{+} u_{r}^{+} \geqslant 0, C \text { constant, } \\
\int\left(u_{r}^{+}\right)^{2}=\beta_{1}^{2} \int\left|\nabla_{\theta} u^{+}\right|^{2}, \quad \int\left|\nabla_{\theta} u^{+}\right|^{2}=\frac{1}{\alpha_{1} r^{2}} \int\left(u^{+}\right)^{2} .
\end{gathered}
$$

It follows that $u_{r}^{+}=c u^{+} / r\left(c=c_{n}>0\right)$; a similar relation holds for $u^{-}$. Thus $u=r^{b} g(\theta)$ if $u \neq 0$. Since $u$ is bounded, $b \geqslant 0$. By nondegeneracy and Lipschitz 
continuity, $b=1$. Thus $u=r g(\theta)$ if $u \neq 0$ and then,

$$
A g+(n-1) g=0 \quad \text { where } g(\theta) \neq 0,
$$

where $A$ is the Laplacian restricted to $\partial B_{1}$. If $n=2$ then $A g=g^{\prime \prime}$ and the assertions easily follow using Lemma 6.2 and Theorem 2.4.

REMARK 6.1. We do not know whether the isoperimetric inequality $\gamma_{1}(E) \geqslant \gamma_{1}\left(E^{*}\right)$ used in the proof of Lemma 5.1 is a strict inequality whenever $E$ is not a spherical cap. If this is indeed the case then Lemma 6.6(ii) is valid for any $n \geqslant 2$. Indeed, from the proof of Lemma 5.1 we then conclude that, for any $r, u_{0}=l_{2}(x \cdot e)^{+}-l_{1}(x \cdot e)^{-}$ on $\partial B_{r}$ where $e=e(r), l_{i}=l_{i}(r)>0$. Setting

$$
\begin{aligned}
f(r) & =l_{1} e \quad \text { if } u_{0}>0, \\
& =l_{2} e \quad \text { if } u_{0}<0,
\end{aligned}
$$

we have $\Delta(x \cdot f(r))=0$ on $\left\{u_{0} \neq 0\right\}$, which gives

$$
\sum x_{i}\left(f_{i}^{\prime \prime}(r)+\frac{n+1}{r} f_{i}^{\prime}(r)\right)=0
$$

where $f=\left(f_{1}, \ldots, f_{n}\right)$. It follows that $f^{\prime \prime}+(n+1) f^{\prime} / r=0$, or $f(r)=C r^{-n-1}+c$ where $C, c$ are constant vectors in any component of $\left\{u_{0} \neq 0\right\}$. Since $u_{0}$ is bounded, $C=0$ and the assertions in (ii) easily follow.

\section{Properties of the free boundary.}

THEOREM 7.1. There exists a positive constant $c \in(0,1)$ such that for any ball $B_{r} \subset \Omega$ with center in $\partial\{u>0\}$

$$
c \leqslant \frac{\mathcal{L}^{n}\left(B_{r} \cap\{u>0\}\right)}{\mathcal{L}^{n}\left(B_{r}\right)} \leqslant 1-c .
$$

Proof. By nondegeneracy there exists a point $y \in \partial B_{r / 2}$ with $u(y) \geqslant c r$. Since $u$ is Lipschitz, $u(x)>0$ in $B_{\kappa r}(y)$, for some small enough $\kappa$. This establishes the left-hand side of (7.1). To obtain the second inequality, let

$$
\begin{aligned}
\Delta v=0 & \text { in } B_{r}, \\
v=u & \text { on } \partial B_{r} .
\end{aligned}
$$

Then $v \geqslant u$ in $B_{r}$ and (cf. the proof of Theorem 2.1)

$$
\int_{B_{r} \cap\{u \leqslant 0<v\}}|\Lambda| \geqslant \int_{B_{r}}|\nabla(u-v)|^{2} \geqslant \frac{c}{r^{2}} \int_{B_{r}}|u-v|^{2} \geqslant \frac{c}{r^{2}} f_{B_{k r}}|u-v|^{2},
$$

for any $0<\kappa<1$.

If $y \in B_{\kappa r}$ then (we take the center of $B_{r}$ to be at the origin)

$$
|v(y)-v(0)| \leqslant|y||\nabla v| \leqslant \kappa r \frac{C}{r} f_{\partial B_{r}}|u| \leqslant C \kappa r,
$$

and $v(0)=f_{\partial B_{r}} v=f_{\partial B_{r}} u,|u(y)| \leqslant C \kappa r$. It follows that $|v(y)-u(y)| \geqslant \int_{\partial B_{r}} u-C \kappa r$. Recalling Theorem 6.3 we obtain

$$
|v(y)-u(y)| \geqslant c r-C \kappa r \geqslant c r / 2
$$


if $\kappa$ is small enough. Using this estimate in (7.2) we find that

$$
\varrho^{n}\left(B_{r} \cap\{u \leqslant 0\}\right) \geqslant \frac{C}{r^{2}} \int_{B_{\mathrm{k} r}} c^{2} r^{2} \geqslant c r^{n} \quad(c>0) .
$$

Since $u^{ \pm}$are continuous and subharmonic, the measures $d \lambda^{+}=\Delta u^{+}$and $d \lambda^{-}=$ $\Delta u^{-}$are Radon measures supported on $\Omega \cap \partial\{u>0\}$ and $\Omega \cap \partial\{u<0\}$, respectively.

THEOREM 7.2. For any $D \Subset \Omega$ there exist positive constants $c, C$ such that, for any $B_{r} \subset D$ with center in $\partial\{u>0\}$,

$$
\begin{gathered}
c r^{n-1} \leqslant \int_{B_{r}} d \lambda^{+} \leqslant C r^{n-1}, \\
\int_{B_{r}} d \lambda^{-} \leqslant C r^{n-1} .
\end{gathered}
$$

Proof. Let $x \in \partial\{u>0\}$. For almost all $r$ with $B_{r}(x) \subset \Omega$,

$$
\int_{B_{r}(x)} d \lambda^{+}=\int_{\partial B_{r}(x)} \nabla u^{+} \cdot \nu d H^{n-1} \leqslant C r^{n-1}
$$

since $u^{+}$is Lipschitz continuous. This proves the second inequality in (7.3). The proof of (7.4) is similar. The proof of the first inequality in (7.3) is similar to the proof given in [1, Theorem 4.3], with $u$ replaced by $u^{+}$.

TheOREM 7.3 (Representative TheOREM). (i) If $D \Subset \Omega$ then

$$
H^{n-1}(D \cap \partial\{u>0\})<\infty .
$$

(ii) There exist Borel functions $q_{u}^{ \pm}$such that

$$
\Delta u^{ \pm}=q_{u}^{ \pm} H^{n-1} L \partial\{u>0\},
$$

that is, for every $\zeta \in C_{0}^{\infty}(\Omega)$,

$$
-\int_{\Omega} \nabla u^{ \pm} \cdot \nabla \zeta=\int_{\Omega \cap \partial\{u>0\}} \zeta q_{u}^{ \pm} d H^{n-1} .
$$

(iii) For any $D \Subset \Omega$ there exist positive constants $c, C$ depending on $D, \Omega$, the constant $c$ in Corollary 3.2 and any bound on $|\nabla u|_{L^{\infty}(D)}$, such that for any ball $B_{r}(x) \subset D$ with $x \in \partial\{u>0\}$,

$$
\begin{aligned}
c & \leqslant q_{u}^{+} \leqslant C, \\
c r^{n-1} & \leqslant H^{n-1}\left(B_{r}(x) \cap \partial\{u>0\}\right) \leqslant C r^{n-1}, \\
0 & \leqslant q_{u}^{-} \leqslant C .
\end{aligned}
$$

Proof. For any compact set $E \subset D \cap \partial\{u>0\}$ and small $r$ choose a covering of $E$ with balls $B_{r}\left(y_{i}\right)$ such that $\sum I_{B_{2 r}\left(y_{i}\right)} \leqslant C$. Choosing $x_{i} \in B_{r}\left(y_{i}\right) \cap E$ we have, by Theorem 7.2,

$$
\sum_{i} r^{n-1} \leqslant C \sum_{i} \lambda^{+}\left(B_{r}\left(x_{i}\right)\right) \leqslant C \lambda^{+}\left(B_{4 r}(E)\right)
$$


which gives

$$
H^{n-1}(E) \leqslant C \lambda^{+}(E) .
$$

Thus (i) holds and $H^{n-1} L(D \cap \partial\{u>0\})$ is absolutely continuous with respect to $\lambda^{+}$.

Next, the support of $\lambda^{+}$is contained in $\partial\{u>0\}$ and, by Theorem 7.2,

$$
\lambda^{+}\left(B_{r}\right) \leqslant C r^{n-1} \text { for any ball } B_{r} \subset D \text {; }
$$

from this it follows that $\lambda^{+}(E) \leqslant C H^{n-1}(E)$. We have thus shown that the Radon measure $\lambda^{+}$is absolutely continuous with respect to the Radon measure

$$
H^{n-1} L \partial\{u>0\}
$$

and vice versa. Setting $q_{u}^{+}=d \lambda^{+} / d H^{n-1} \mathcal{L}(\partial\{u>0\})$ we see that (7.5) holds (for $\left.\Delta u^{+}\right)$, and (7.10) and (7.11) establish (7.7) and (7.8).

Using the assertion (i) we can now proceed with proving (ii) and (iii) for $\lambda^{-}$by the same proof as for $\lambda^{+}$.

Since $\partial\{u>0\}$ has finite $H^{n-1}$ measure, the set $A=\Omega \cap\{u>0\}$ has finite perimeter locally in $\Omega$, that is, $\mu_{u} \equiv-\nabla I_{A}$ is a Borel measure and the total variation $\left|\mu_{u}\right|$ is a radon measure. We denote by $\partial_{\text {red }}\{u>0\}$ the reduced boundary of $\partial\{u>0\}$.

TheOrem 7.4 (IDENTIFICATION TheOrem). Let $x_{0} \in \partial_{\text {red }}\{u>0\}$ with

$$
\begin{gathered}
\theta^{* n-1}\left(H^{n-1} L \partial\{u>0\}, x_{0}\right) \leqslant 1, \\
\int_{B_{r}\left(x_{0}\right) \cap \partial\{u>0\}}\left|q_{u}^{ \pm}-q_{u}^{ \pm}\left(x_{0}\right)\right|=o(1), \quad r \rightarrow 0 .
\end{gathered}
$$

(i) If $\gamma>0$ (in Corollary 6.5) and $n=2$, then

$$
u\left(x_{0}+x\right)=\mu_{2}\left(x \cdot e\left(x_{0}\right)\right)^{+}-\mu_{1}\left(x \cdot e\left(x_{0}\right)\right)^{-}+o(|x|) \text { as }|x| \rightarrow 0,
$$

where $\mu_{i}>0, \mu_{1}^{2}-\mu_{2}^{2}=\Lambda q^{2}\left(x_{0}\right)$, and

$$
\left(\mu_{2}-\mu_{1}\right) e\left(x_{0}\right)=\left(q_{u}^{+}\left(x_{0}\right)-q_{u}^{-}\left(x_{0}\right)\right) \nu_{u}\left(x_{0}\right) \text {. }
$$

(ii) If $\gamma=0$, then

$$
u\left(x_{0}+x\right)=q_{u}^{+}\left(x_{0}\right) \max \left\{-x \cdot \nu_{u}\left(x_{0}\right), 0\right\}+o(|x|)
$$

as $|x| \rightarrow 0$, and $\left(q_{u}^{+}\left(x_{0}\right)\right)^{2}=\left(\lambda_{2}^{2}-\lambda^{2}(0)\right) q^{2}\left(x_{0}\right)$.

Here $\nu_{u}\left(x_{0}\right)$ is the outward normal to $\partial\{u>0\}$ at $x_{0}$.

Proof. Take a blow-up sequence $u_{\varepsilon}(x)=u\left(x_{0}+\varepsilon x\right) / \varepsilon$ with $u_{\varepsilon} \rightarrow u_{0}$ uniformly in compact subsets. Then $\Delta u_{\varepsilon} \rightarrow \Delta u_{0}$ as distributions, and thus also as measures. From (7.5) we deduce that

$$
\Delta u_{\varepsilon}^{ \pm}=q_{u}^{ \pm}\left(x_{0}+\varepsilon x\right) H^{n-1} L \partial\left\{u^{\varepsilon}>0\right\} .
$$

If $\gamma>0$ and $n=2$ then, by Lemma 6.6,

$$
u_{0}=\mu_{2}(x \cdot e)^{+}-\mu_{1}(x \cdot e)^{-} \quad(e \text { constant vector })
$$


and therefore,

$$
\Delta u_{0}^{+}-\Delta u_{0}^{-}=\left(\mu_{1}-\mu_{2}\right) e d H^{n-1} L \Pi_{e}
$$

where $\Pi_{e}$ is the hyperplane orthogonal to $e$. We thus conclude that

$$
\left[q_{u}^{+}\left(x_{0}+\varepsilon x\right)-q_{u}^{-}\left(x_{0}+\varepsilon x\right)\right] d H^{n-1} L \partial\left\{u^{\varepsilon}>0\right\} \rightarrow\left(\mu_{1}-\mu_{2}\right) e d H^{n-1} L \Pi_{e} .
$$

Since $x_{0} \in \partial_{\text {red }}\{\mathrm{u}>0\}$ we have [10, Theorem 3.7]

$$
d H^{n-1} L \partial\left\{u^{\varepsilon}>0\right\} \rightarrow d H^{n-1} L \Pi_{0}
$$

where $\Pi_{0}=\left\{x ; \nu_{u}\left(x_{0}\right) \cdot x=0\right\}$. Recalling (7.12) we deduce that

$$
\left(q_{u}^{+}\left(x_{0}\right)-q_{u}^{-}\left(x_{0}\right)\right) d H^{n-1} L \Pi_{0}=\left(\mu_{2}-\mu_{1}\right) e d H^{n-1} L \Pi_{e}
$$

so that

$$
\left(\mu_{2}-\mu_{1}\right) e=\left(q_{u}^{+}\left(x_{0}\right)-q_{u}^{-}\left(x_{0}\right)\right) \nu_{u}\left(x_{0}\right) ;
$$

also $\mu_{1}^{2}-\mu_{2}^{2}=\Lambda q^{2}\left(x_{0}\right)$. Thus the $\mu_{i}$ and $e$ are uniquely determined, independently of the blow-up sequence, and assertion (i) follows.

Consider next the case $\gamma=0$. By Lemma 6.6 we then have $u_{0} \geqslant 0$ for any blow-up limit of the $u_{\varepsilon}$. We can then proceed as in Theorem 4.8 of [1]. Thus, taking $\nu_{u}\left(x_{0}\right)=e_{n}$, the proof that $u_{0}>0$ if $x_{n}<0, u_{0}=0$ if $x_{n}>0$ is the same as in [1]. Next, setting

$$
\mu_{w}=-\nabla I_{(\Omega \cap\{w>0\})}
$$

for any function $w$ for which $\partial\{w>0\}$ has finite $H^{n-1}$ measure, we have, for every compact subset $E \subset B_{r}^{\prime}\left(B_{r}^{\prime}\right.$ is the ball in $\left.R^{n-1}\right)$,

$$
\begin{aligned}
H^{n-1}(E) & =\mu_{u_{\varepsilon}^{+}}(\operatorname{Ex}(-1,1)) \cdot e_{n} \leqslant H^{n-1}\left(\partial\left\{u_{\varepsilon}^{+}>0\right\} \cap(\operatorname{Ex}(-1,1))\right) \\
& =H^{n-1}\left(\partial\left\{u_{\varepsilon}>0\right\} \cap(E x(-1,1))\right)
\end{aligned}
$$

and we can again proceed as in [1], thereby establishing that $u_{0}(x)=u_{0}^{+}(x)=$ $-q_{u}\left(x_{0}\right) x_{n}$ if $x_{n}<0$, and the proof of (ii) thereby follows; the last assertion in (ii) follows from Lemma 6.2 and Remark 2.1.

REMARK 7.1. From Theorem 7.1 it follows (by $[7,4.5 .6(3)]$ ) that

$$
H^{n-1}\left(\partial\{u>0\} \backslash \partial_{\text {red }}\{u>0\}\right)=0 .
$$

From [7, 4.5.6(2), 2.9.8 and 2.9.9] applied to $H^{n-1} L \partial\{u>0\}$ and the Vitali relation $\left\{\left(x, B_{r}(x)\right): x \in \partial\{u>0\}\right.$ and $\left.B_{r}(x) \subset \Omega\right\}$ it follows that for $H^{n-1}$ a.a. $x_{0} \in$ $\partial_{\text {red }}\{u>0\}$ the assumptions (7.12) and (7.13) are satisfied. Thus Theorem 7.4 shows that for $H^{n-1}$ a.a. $x \in \partial\{u>0\}$ the free boundary in a neighborhood of $x_{0}$ is approximately a hyperplane.

REMARK 7.2. In special models arising in jet flows $[5,6]$ it has been shown that the free boundary is a continuous graph. In the next section we prove, more generally, that the free boundary is $C^{1}$ if $n=2$.

8. Differentiability of the free boundary $(n=2)$. In this section we prove that, in case $n=2$, the free boundary is continuously differentiable. The first lemma is valid for any $n \geqslant 2$. In proving it we shall use the fact that

$$
\text { the sets }\{u>0\} \text { and }\{u<0\} \text { are connected to the boundary of } \Omega \text {. }
$$


To show this, suppose $K$ is a component of $\{u>0\}$ which is not connected to the boundary. Then, by replacing $u$ in $K$ by 0 we obtain a new function $\tilde{u}$ with smaller functional $J(\tilde{u})$, which is a contradiction.

LEMMA 8.1. If $u$ and $\tilde{u}$ are both minimizers of $J$ in a bounded domain $D$, and if $\tilde{u}>u$ on $\partial D$, then $\tilde{u}>u$ in $\{u \neq 0\}$.

Proof. Set $v_{1}=\min \{u, \tilde{u}\}$ and $v_{2}=\max \{u, \tilde{u}\}$. Then $v_{1}=u$ on $\partial D$ and therefore, $J\left(v_{1}\right) \geqslant J(u)$. Similarly, $v_{2}=\tilde{u}$ on $\partial D$ and therefore, $J\left(v_{2}\right) \geqslant J(\tilde{u})$. However, $J\left(v_{1}\right)+J\left(v_{2}\right)=J(u)+J(\tilde{u})$ as seen by writing explicitly the terms in each $J$. It follows that $J\left(v_{1}\right)=J(u)$.

Suppose $u\left(x^{0}\right)=\tilde{u}\left(x^{0}\right) \neq 0$ and $u-\tilde{u}$ changes sign in any neighborhood of $x^{0}$. Then $v_{1}$ is not harmonic in any neighborhood of $x^{0}$. We introduce the function $w$ defined by

$$
\begin{aligned}
\Delta w=0 & \text { in } B_{r}\left(x^{0}\right), \\
w=v_{1} & \text { on } \partial B_{r}\left(x^{0}\right)
\end{aligned}
$$

for some small $r>0$, and $w=v_{1}$ in $D \backslash B_{r}\left(x^{0}\right)$. By the Dirichlet principle we find that $J(w)<J\left(v_{1}\right)=J(u)$, contradicting the minimality of $u$. Thus we conclude that either $\tilde{u} \geqslant u$ or $u \geqslant \tilde{u}$ in some neighborhood of $x^{0}$. Starting with $x^{0}$ near $\partial D$ and recalling (8.1), we deduce that $\tilde{u} \geqslant u$ on the set $\{u \neq 0\}$; furthermore, by the strong maximum principle, $\tilde{u}>u$ in this set.

From now on we make the assumptions

$$
n=2, \quad q(x) \equiv 1 .
$$

For definiteness we shall also assume that $\Lambda<0$. We denote points in $R^{2}$ by $X$ or $(x, y)$. Set $e_{1}=(1,0)$ and $e_{2}=(0,1)$.

Lemma 8.2. For any $\varepsilon_{0}>0, \eta>0$ there is $a \delta=\delta\left(\varepsilon_{0}, \eta\right)>0$ such that for any minimizer $u$ in the rectangle $I=\{-3<x<3,-1<y<1\}$ satisfying

(i) the free boundary contains $(0,0)$ and lies in the strip $\{|y|<\delta\}$,

(ii) $u(A)<-\eta$ where $A=\left(0,-\frac{1}{2}\right)$,

the free boundary in $I_{0}=\{-1<x<1,-1<y<1\}$ is a graph in any direction $\varepsilon e_{2} \pm e_{1}, \varepsilon \geqslant \varepsilon_{0}$.

Proof. Take a circle $K_{\mu}^{1}:(x+2)^{2}+(y-\mu)^{2}<\delta^{-3 / 2}$ with center $(-2, \mu)$ and radius $\delta^{-3 / 4}$ and increase $\mu$ from $-\infty$ until, at $\mu=\mu_{1}, \partial K_{\mu_{1}}^{1}$ touches the free boundary of $u$ for the first time. Since $\partial K_{\mu_{1}}^{1} \cap\{x=-2\}$ lies in $\{y<\delta\}$,

$$
\partial K_{\mu_{1}}^{1} \cap\left\{-3<x<-\frac{5}{2}\right\} \text { and } \partial K_{\mu_{1}}^{1} \cap\left\{-\frac{3}{2}<x<3\right\}
$$

both lie below $y=\delta-C \delta^{3 / 4}$ and thus, also below $y=-\delta$ if $\delta$ is small enough. Consequently, $\partial K_{\mu_{1}}^{1} \cap \partial\{u>0\}$ lies in $\left\{-\frac{5}{2}<\mathrm{x}<-\frac{3}{2}\right\}$ and contains a point $E_{1}=$ $\left(x_{1}, y_{1}\right)$ with $-\frac{5}{2}<x_{1}<-\frac{3}{2},-\delta<y_{1}<\delta$.

Similarly, we construct a circle $K_{\mu_{2}}^{2}$ whose closure intersects the free boundary only at points of $\partial K_{\mu_{2}}^{2}$ lying in $\left\{\frac{3}{2}<x<\frac{5}{2}\right\}$, and a point $E_{2}=\left(x_{2}, y_{2}\right)$ on $\partial K_{\mu_{2}}^{2} \cap$ $\partial\{u>0\}$, with $\frac{3}{2}<x_{2}<\frac{5}{2},-\delta<y_{2}<\delta$; further, $K_{\mu_{1}}^{1} \cap\{|y| \leqslant \delta\}$ and $K_{\mu_{2}}^{2} \cap\{|y| \leqslant$ $\delta\}$ are disjoint. We denote by $\sigma$ the curve consisting of (i) three line segments on 
$y=-\delta$, from $(-3,-\delta)$ to the left endpoint of $\{y=-\delta\} \cap \partial K_{\mu_{1}}^{1}$, from the right endpoint of $\{y=-\delta\} \cap K_{\mu_{1}}^{1}$ to the left endpoint of $\{y=-\delta\} \cap \partial K_{\mu_{2}}^{2}$ and from the right endpoint of $(y=-\delta) \cap \partial K_{\mu_{2}}^{2}$ to $(3,-\delta)$, and (ii) the arcs of $\partial K_{\mu_{1}}^{i}$ lying in $\{|y|<\delta\}$.

Denote by $\Sigma_{-}$the part of $I$ lying below $\sigma$. Notice that $u<0$ in $\Sigma_{-}$.

From assumption (ii) and Harnack's inequality we get

$$
u(X) \leqslant-c \eta \operatorname{dist}(X, \sigma) \text { if } X \in \Sigma_{-}(c>0) .
$$

We next claim

there exists a $C^{1}$ curve $\sigma_{i}: y=f_{i}(x)$ in $I$ such that $E_{i} \in \sigma_{i}$ and $u>0$ above $\sigma_{i}$ (in $I$ ), for $i=1,2$; furthermore, $f_{i}^{\prime}(x)-f_{i}^{\prime}\left(x_{i}\right)$

$\rightarrow 0$ as $x-x_{i} \rightarrow 0$, uniformly with respect to $u$.

Notice that $\sigma$ and $E_{i}$ depend on $u$ and so does $f_{i}$. To prove (8.4) suppose first that there exist sequences $E_{1}=E_{1}(m)=\left(x_{1}(m), y_{1}(m)\right), u=u_{m}$ and $Z_{m}=\left(\tilde{x}_{m}, \tilde{y}_{m}\right)$ with $u_{m}\left(Z_{m}\right) \leqslant 0$, such that $\left|Z_{m}-E_{1}(m)\right| \rightarrow 0$ and the angle between $\overrightarrow{E_{1}(m) Z_{m}}$ and the tangent to $\sigma$ at $E_{1}(m)$ does not converge to zero as $m \rightarrow \infty$. Set $r_{m}=\left|Z_{m}-E_{1}(m)\right|$ and consider a blow-up sequence of $u_{m}$ with respect to $B_{r_{m}}\left(E_{1}(m)\right)$. Let $w$ be a blow-up limit. We can rotate the coordinates in such a way that

$$
w(x, y) \leqslant 0 \text { if } y \leqslant 0,
$$

and then $w\left(x_{0}, y_{0}\right) \leqslant 0$ for some point $\left(x_{0}, y_{0}\right)$ with $y_{0}>0$. Consequently, $w$ is not a 2-plane solution.

In view of (8.3) and the assumption $\Lambda<0, w$ does have two phases.

By Corollary 6.5 and Lemma 6.6,

$$
w(X)=\alpha y+o(|X|) \quad \text { if } y<0,|X| \rightarrow 0
$$

where $\alpha$ is determined by $\alpha^{2}\left(\alpha^{2}+|\Lambda|\right)=\gamma, \gamma=\lim _{r \rightarrow 0} \psi(r)$, where

$$
\psi(r)=\frac{1}{r^{4}} \int_{B_{r}}\left|\nabla w^{+}\right|^{2} \cdot \int_{B_{r}}\left|\nabla w^{-}\right|^{2} .
$$

Similarly, working with blow-up sequences $\frac{1}{m} w(m X)(m \rightarrow \infty)$ we find that

$$
w(X)=\beta y+o(|X|) \text { if } y<0,|X| \rightarrow \infty
$$

where $\beta^{2}\left(\beta^{2}+|\Lambda|\right)=\gamma_{0}, \gamma_{0}=\lim _{r \rightarrow \infty} \psi(r)$. Since, by (8.6), $w$ is not a 2-plane solution, Lemma 6.6 shows that $\gamma_{0}>\gamma$ and, consequently,

$$
\beta>\alpha \text {. }
$$

Let $\Omega_{R}=\{w<0\} \cap B_{R}$. If we formally apply Green's formula to $w$ and $G=$ $y /\left(x^{2}+y^{2}\right)-y / R^{2}$ in $\Omega_{R} \backslash B_{\varepsilon}$, we obtain

$$
\int_{\partial \Omega_{R} \backslash B_{\varepsilon}}\left(G w_{\nu}-w G_{\nu}\right)+\int_{\partial B_{\varepsilon} \cap\{w<0\}}\left(G w_{\nu}-w G_{\nu}\right)=0
$$


where $\nu$ is the inner normal. In order to justify (8.10) and make sense of the integrals over the free boundary we apply (7.6) with $u^{-}=w^{-}$and $\zeta=\eta G$ where $\eta=\eta(r)$ is given by

$$
\eta(r)= \begin{cases}1 & \text { if } r \leqslant R \\ 1-(r-R)^{2} / \delta^{2} & \text { if } R<r<R+\delta, \\ 0 & \text { if } r>R+\delta,\end{cases}
$$

and then let $\delta \rightarrow 0$. We then obtain (8.10) with $\int G_{\nu} w=0$ on the free boundary and $\int G w_{\nu}=-\int q_{w}^{-} G d H^{1}$ on the free boundary. By (8.5), $G \geqslant 0$ on the free boundary and therefore the last integral is nonnegative. We thus conclude from (8.10) that

$$
\int_{\partial B_{r} \cap\{w<0\}} w G_{\nu} \leqslant \int_{\partial B_{\varepsilon} \cap\{w<0\}}\left(G w_{\nu}-w G_{\nu}\right) .
$$

Using (8.8) we compute that

$$
\int_{\partial B_{R} \cap\{w<0\}} w G_{\nu}=\int 2 \frac{\sin \theta}{R^{2}}(\beta y+o(R))=2 \beta \int \sin ^{2} \theta d \theta+\eta(R)
$$

where $\eta(R) \rightarrow 0$ if $R \rightarrow \infty$. Similarly,

$$
-\int_{\partial B_{\varepsilon} \cap\{w<0\}} w G_{\nu}=\alpha \int \sin ^{2} \theta d \theta+\eta_{0}(\varepsilon)
$$

where $\eta_{0}(\varepsilon) \rightarrow 0$ if $\varepsilon \rightarrow 0$. Finally, for a sequence $\varepsilon_{i} \rightarrow 0$ we have

$$
\int_{\partial B_{\varepsilon_{i}} \cap\{w<0\}} G w_{\nu}=\alpha \int \sin ^{2} \theta d \theta+\eta_{1}\left(\varepsilon_{i}\right)
$$

with $\eta_{1}\left(\varepsilon_{i}\right) \rightarrow 0$ if $\varepsilon_{i} \rightarrow 0$. Indeed, this follows from

$$
\begin{aligned}
\frac{1}{\bar{\varepsilon}} \int_{0}^{\bar{\varepsilon}} d \varepsilon \int G w_{\nu} d s & =\frac{1}{\bar{\varepsilon}} \int \sin \theta[w]_{r=\bar{\varepsilon}} d \theta+\frac{o(\bar{\varepsilon})}{\bar{\varepsilon}} \\
& =\alpha \int \sin ^{2} \theta d \theta+\frac{o(\bar{\varepsilon})}{\bar{\varepsilon}} .
\end{aligned}
$$

Using the preceding estimates in (8.11) we get

$$
2 \alpha \int_{\pi+o(1)}^{2 \pi+o(1)} \sin ^{2} \theta \geqslant 2 \beta \int_{\pi}^{2 \pi} \sin ^{2} \theta+o(1)
$$

where $o(1) \rightarrow 0$ if $\varepsilon \rightarrow 0, R \rightarrow \infty$; this contradicts (8.9).

We have thus proved that there cannot exist sequences $E_{1}(m), Z_{m}, u_{m}$ as above. It follows that, for each $u,\{u<0\} \cap\left\{x>x_{1}\right\}$ lies below a polygonal curve $\pi_{0}$ with sides $\overline{Z^{m} Z^{m+1}}$ having slope $\phi_{m}$ which decreases to the slope $\phi_{\infty}$ of $\sigma$ at $E_{1}$, uniformly with respect to $u$, as $\left|Z^{m}-E_{1}\right| \rightarrow 0$. We modify $\pi_{0}$ near its vertices so as to obtain a $C^{1}$ curve $y=f_{1}(x)$ lying above $\pi_{0}$ with slope converging to $\phi_{\infty}$ as $x \downarrow x_{1}$. Similarly, we can construct $y=f_{1}(x)$ for $x<x_{2}$, and this completes the construction of $\sigma_{1}$ as asserted in (8.4). $\sigma_{2}$ is constructed similarly.

REMARK 8.1. The assertion (8.4) remains valid also if condition (ii) is dropped. Indeed, if in the previous proof $w$ is a 2-phase solution, then the proof is the same. If, on the other hand, $w$ is a 1 -phase solution (and then $w \geqslant 0$ since $\Lambda<0$ ) then we 
get a contradiction to Lemma 8.4 below; Leinma 8.4 is proved independently of Lemmas 8.1-8.3. This remark will be used in proving Lemma 8.11 (which is an extension of Lemma 8.2 to the case where condition (ii) is dropped).

Now consider in the strip $I^{\delta}=I \cap\{|y|<\sqrt{\delta}\} \cap\left\{x_{1}<x<x_{2}\right\}$ the quotient difference $\Delta_{h, l} u$ of $u$ in the direction $l$ of $\varepsilon e_{2} \pm e_{1}$, with increment $h$, where $0<h<2 \delta$, i.e.,

$$
\Delta_{h, l} u(X)=(u(X+h l)-u(X)) / h .
$$

We claim that

$$
\Delta_{h, l} u \geqslant c>0
$$

in $I^{\delta}$. We first prove (8.12) on $y=\sqrt{\delta}$. If the assertion is not true then for sequences $u_{m}, X_{m}=\left(x_{m}, \delta_{m}^{1 / 2}\right)$ with $\delta_{m} \rightarrow 0$ there holds $\Delta_{h_{m}, l_{m}} u_{m}\left(X_{m}\right) \rightarrow 0$, with $0<h_{m}<2 \delta_{m}$ and $l_{m} \rightarrow l, l$ in direction $\varepsilon e_{2} \pm e_{1}, \varepsilon \geqslant \varepsilon_{0}$. Take a blow-up about free boundary points of $u_{m}$ on $\left\{x=x_{m}\right\}$ with radii $\leqslant 2 \delta_{m}^{1 / 2}$. Since the free boundary of $u_{m}$ lies in $\left\{|y|<\delta_{m}\right\}$, the blow-up limit $w$ is a 2-plane solution (we use here (8.3) and the assumption $\Lambda<0$ ) and its free boundary is the $x$-axis. Since

$$
\Delta_{h_{m}, l_{m}} u_{m}\left(X_{m}\right) \rightarrow \partial w\left(X_{0}\right) / \partial l
$$

where $X_{0}=(0,1)$ and $\varepsilon \neq 0$, we get a contradiction.

Similarly, we can establish $(8.12)$ on $y=-\sqrt{\delta}$. Consider now the quotient difference on the vertical line $V_{1}$ of $\partial I^{\delta}$ passing through $E_{1}$. If (8.12) does not hold on $V_{1}$, say

$$
\Delta_{h_{m}, l_{m}} u_{m}\left(X_{m}\right) \rightarrow 0 \quad\left(X_{m} \in V_{1}\right),
$$

then we make a blow-up about $E_{1}$ with radii $r_{m}=\max \left\{h_{m},\left|X_{m}-E_{1}\right|\right\}$. Recalling that near $E_{1}$ the free boundary lies between $\sigma$ and $\sigma_{1}$ and using (8.4), we again deduce that the blow-up limit $w$ is a 2-plane solution, with the $x$-axis as the free boundary; Further,

$$
\begin{array}{ll}
\frac{\partial w}{\partial l}\left(X_{0}\right)=0 & \text { if } h_{m}=o\left(\left|X_{m}-E_{1}\right|\right), \\
\Delta_{h_{0}, l_{w}}\left(X_{0}\right)=0 & \text { if }\left|h_{m}\right| \geqslant c_{0}\left|X_{m}-E_{1}\right|\left(c_{0}>0\right)
\end{array}
$$

for some $h_{0}, X_{0}$ and some $l$ in direction $\varepsilon e_{2} \pm e_{1}, \varepsilon \geqslant \varepsilon_{0}$. But this is impossible since $w$ is a function of $y$ only.

Having proved (8.12) on $\partial I^{\delta}$ we now translate $u$ in the direction $l$ by considering

$$
u_{\tau}(X)=u(X+\tau l), \quad \tau>0 .
$$

In view of (8.12), $u_{\tau}>u$ on $\partial I^{\delta}$ if $0<\tau<2 \delta$. Since $q(x) \equiv 1, u_{\tau}$ is a minimizer for the same functional $J$ as $u$. Appealing to Lemma 8.1 we conclude that $u_{\tau}>u$ in $\{u \neq 0\}$, from which the assertion follows.

LeMMA 8.3. Any global minimizer $u$ with two phases must be a 2-plane solution.

Proof. For a sequence $m \rightarrow \infty$ we have $u_{m}(X) \equiv u(m X) / m \rightarrow v(X)$ where $v(X)$ is a 2-plane solution. Indeed,

$$
\psi(r) \equiv \frac{1}{r^{4}} \int_{B_{r}}\left|\nabla u^{-}\right|^{2} \int_{B_{r}}\left|\nabla u^{+}\right|^{2} \uparrow \gamma \quad \text { as } r \uparrow \infty
$$


and since $\psi\left(r_{0}\right)>0$ for some $r_{0}>0$ (since $u$ has two phases) it follows that $\gamma>0$. On the other hand, $v$ satisfies (6.13) and, by Lemma 6.6(ii),

$$
v(x, y)= \begin{cases}\mu_{1} y & \text { if } y>0, \\ \mu_{2} y & \text { if } y<0,\end{cases}
$$

where $\mu_{1}>0, \mu_{2}>0, \mu_{1}^{2} \mu_{2}^{2}=\gamma$.

Given $\varepsilon_{0}>0$ and $\eta=\mu_{2} / 2$, if $m$ is large enough then the $u_{m}$ restricted to $I$ satisfy the conditions of Lemma 8.2 (recall that the lemma is valid uniformly with respect to the class of all minimizers $u$ ). Hence, the free boundary $\partial\left\{u_{m}>0\right\}$ (for $m \geqslant m\left(\varepsilon_{0}\right)$ ) in $I_{0}$ is a graph in the direction $( \pm 1, \varepsilon)$ for any $\varepsilon \geqslant \varepsilon_{0}$. It follows that $\partial\{u>0\} \cap$ $\{|x|<m,|y|<m\}$ is a graph in any direction $( \pm 1, \varepsilon)$ where $\varepsilon \geqslant \varepsilon_{0}$. Since $\varepsilon_{0}$ can be chosen arbitrarily small (and $\left.m \geqslant m\left(\varepsilon_{0}\right)\right), \partial\{u>0\}$ must coincide with the $x$-axis. By uniqueness for the Cauchy-Kowalewski theorem $u$ is thus linear in $y$ for $y>0$ and for $y<0$.

LEMMA 8.4. Any global minimizer $u$ with one phase must be a 1-plane solution.

Naturally, to exclude a trivial case we assume that $u \geqslant 0$ in $R^{2}$ with (say) $0 \in \partial\{u>0\}$ and with $\lambda>0$, where $J(u)=\int\left(|\nabla u|^{2}+\lambda^{2} I_{\{u>0\}}\right)$.

Proof. The function $|\nabla u|$ is subharmonic and $|\nabla u|=\lambda$ on $\partial\{u>0\}$. Proceeding as in [2] (see also [9, p. 327]) we deduce that $|\nabla u|$ takes its maximum on the free boundary and, consequently, the free boundary is convex to $\{u>0\}$. If $\partial\{u>0\}$ is not a straight line then the blow-up limit of a subsequence of $u_{m}(X)=u(m X) / m$ converges to a minimizer $v$ whose free boundary includes two rays forming an angle $\neq \pi$ at the origin; this contradicts the Cauchy-Kowalewski theorem since $u=0$, $\partial u / \partial \nu=0$ on each of these rays.

LEMMA 8.5. For any $\gamma>0$ and $C_{0}>0$ there is $a \delta=\delta\left(\gamma, C_{0}\right)$ such that if $u$ is a minimizer in $B_{1}$ with $|\nabla u| \leqslant C_{0}$ then for any ball $B_{\delta}\left(X^{0}\right) \subset B_{1 / 2}$ with center in the free boundary, the $\gamma$-flatness condition holds, i.e., the free boundary of $u$ in $B_{\delta}\left(X^{0}\right)$ lies in a strip with center $X^{0}$ and width $2 \gamma$.

Proof. If the assertion is not true then there is a sequence $B_{\delta_{m}}\left(X_{m}\right) \subset B_{1 / 2}$ with $\delta_{m} \rightarrow 0$ such that the flatness condition does not hold for some $u_{m} ; X_{m} \in \partial\left\{u_{m}>0\right\}$. A blow-up sequence with respect to $B_{\delta_{m}}\left(X_{m}\right)$ is convergent to a minimizer $v$ in $R^{2}$ and the free boundary of $v$ in $B_{1}(0)$ does not lie in a $(2 \gamma)$-strip with 0 in the centerline of the strip. If $v$ has two phases, this contradicts Lemma 8.3, whereas if $v$ has one phase, Lemma 8.4 is contradicted.

LEMMA 8.6. If $u$ satisfies the $\gamma$-flatness condition in $B_{1}=B_{1}(0)$ in direction $(0,1)$ and if

$$
u(A)>M u(P) \text { where } A=\left(0, \frac{1}{2}\right), P \in\{u>0\} \cap B_{1 / 2},
$$

then, for some absolute constant $C, \operatorname{dist}(P, \partial\{u>0\})<2 \gamma+C / M$.

Proof. By the flatness assumption $u>0$ in $B_{1} \cap\{y>\varepsilon / 2\}$ for any $\varepsilon>2 \gamma$. Suppose $\operatorname{dist}(P, \partial\{u>0\})>\varepsilon$; then also $\operatorname{dist}(P,\{y<\varepsilon / 2\})>\varepsilon / 2$. Applying Harnack's inequality in $B_{1} \cap\{y>\varepsilon / 2\}$ we get $u(P)>c \varepsilon u(A)$. Hence, by (8.13), $1 / M>c \varepsilon$, i.e., $\varepsilon<1 / c M$. 
Lemma 8.7. For $\gamma$ sufficiently small let $\delta=\delta\left(\gamma, C_{0}\right)$ be as in Lemma 8.5, and let $B_{\delta}\left(X_{0}\right)$ be any ball in $B_{1 / 2}$ with $X_{0}$ for which the $\gamma$-flatness holds in the direction $(0,1)$, say, and $u(A)>0$ where $A=X_{0}+(0, \delta / 2)$. Then

$$
u(A) \geqslant \gamma \sup _{B_{\delta / 2}\left(X_{0}\right)} u \text {. }
$$

Proof. Take, for simplicity, $X_{0}=0$ and normalize by taking $\delta=1$. Set $A_{0}=A$. If the assertion (8.14) is not true then there exists a point $P_{0} \in B_{1 / 2} \cap\{u>0\}$ such that

$$
u\left(P_{0}\right)>\frac{1}{\gamma} u\left(A_{0}\right)
$$

By Lemma 8.6

$$
\operatorname{dist}\left(P_{0},\{y>\gamma\}\right)<C_{0} \gamma .
$$

Let $E$ be a point on the free boundary with

$$
\left|E-P_{0}\right|<\left(C_{0}+2\right) \gamma \text {. }
$$

By the $\gamma$-flatness about $E$, the direction of flatness $\nu_{E}$ at $E$ differs from the direction $(0,1)$ by at most $C \gamma$.

We fix $\eta$ small, to be determined later (independently of $\gamma$ ) and take $\gamma \ll \eta$. By Harnack's inequality in $B_{1} \cap\{y>\eta / 2\}$ we have

$$
\frac{1}{N} u\left(A_{0}\right) \leqslant u(X) \leqslant N u\left(A_{0}\right) \quad \text { if } X \in B_{1 / 2} \cap\{y>\eta\}
$$

where $N=N(\eta)$. Denoting by $G$ the Green function for $-\Delta$ in $\tilde{B} \equiv B_{1 / 4}(E) \cap\{y>$ $-2 \gamma\}$, we can represent the subharmonic function $u^{+}$at $P_{0}$ in the form

$$
u\left(P_{0}\right)=-\int_{\partial \tilde{B}} \frac{\partial G}{\partial \nu} u^{+}=-\int_{S}-\int_{T}
$$

where $S=\partial B_{1 / 4}(E) \cap\{y>\eta\}$ and $T=\partial B_{1 / 4}(E) \cap\{y<\eta\} \cap\left\{(X-E) \cdot \nu_{E}>\right.$ $\gamma\}$ (notice that $u^{+}=0$ on $\partial \tilde{B} \cap\left\{(X-E) \cdot \nu_{E} \leqslant \gamma\right\}$ and, in particular, on $\partial \tilde{B} \cap\{y$ $=-2 \gamma\}$ ). Setting $\sigma(\eta)=\operatorname{meas}(T)$, we have $\sigma(\eta) \rightarrow 0$ if $\eta \rightarrow 0$.

By (8.17), $-\partial G\left(P_{0}, X\right) / \partial \nu \leqslant C \gamma$ if $X \in S \cup T$. Consequently, $-\int_{T} \leqslant$ $C \gamma \delta(\eta) \sup _{T} u^{+}$and (using (8.18))

$$
-\int_{S} \leqslant N u\left(A_{0}\right)(1-\sigma(\eta)) C \gamma .
$$

Recalling (8.15) we conclude that

$$
\frac{1}{\gamma} u\left(A_{0}\right) \leqslant C u\left(P_{0}\right) \leqslant N C \gamma u\left(A_{0}\right)+C \gamma \sigma(\eta) \sup _{T} u^{+} .
$$

Choosing $\eta$ such that $2 C \sigma(\eta)<1$ we find that, provided $N C \gamma / 2 \gamma$, there holds $u\left(A_{0}\right) / \gamma^{2} \leqslant \sup _{T} u^{+}$. Thus, there is a point $P_{1} \in T$ such that

$$
u\left(P_{1}\right)>\frac{1}{\gamma^{2}} u\left(A_{0}\right) \text {. }
$$


Let $A_{1}$ be the point in $B_{1 / 2}(E)$ such that $\overrightarrow{A_{1} E}$ is in the direction $-\nu_{E}$, with $\left|A_{1}-E\right|=1 / 8$. Then, by Harnack's inequality,

$$
u\left(A_{1}\right) \leqslant N u\left(A_{0}\right)<\frac{1}{\gamma} u\left(A_{0}\right)
$$

with the same $N$ as before (if $\eta$ is small enough). The previous setting for $A_{0}, P_{0}$ occurs also for $A_{1}, P_{1}$ since, by (8.19) and (8.20),

$$
u\left(P_{1}\right)>\frac{1}{\gamma^{2} N} u\left(A_{1}\right)>\frac{1}{\gamma} u\left(A_{1}\right) .
$$

We can now repeat the previous proof with $0, A_{0}, P_{0}$ replaced by $E, A_{1}, P_{1}$ and $B_{1 / 2}(0)$ replaced by $B_{1 / 4}(E)$. Thus there is a triple $E_{2}, A_{2}, P_{2}$ such that

$$
u\left(P_{2}\right)>\frac{1}{\gamma} \frac{1}{\gamma^{2} N} u\left(A_{1}\right)
$$

and $u\left(P_{2}\right)>u\left(A_{2}\right) / \gamma>0, E_{2} \in \partial\{u>0\}, \overrightarrow{E_{2} A_{2}}$ is in the direction $\nu_{E_{2}}$ of $\gamma$-flatness at $E_{2},\left|A_{2}-E_{2}\right|=\frac{1}{2} \cdot \frac{1}{4}$.

Continuing in this way, step by step, we construct a sequence $\left(E_{n}, A_{n}, P_{n}\right)$ such that

$$
u\left(P_{n}\right)>\frac{1}{\gamma^{n+1}} \frac{1}{N^{n-1}} u\left(A_{n-1}\right)
$$

and $u\left(P_{n}\right)>u\left(A_{n}\right) / \gamma>0, u\left(E_{n}\right)=0, \overrightarrow{E_{n} A_{n}}$ is in the direction $\nu_{E_{n}}$ of $\gamma$-flatness about $E_{n},\left|A_{n}-E_{n}\right|=\frac{1}{2} 2^{-n}$. Recall that, by Harnack's inequality, $u\left(A_{1}\right)>u\left(A_{0}\right) / N$. Since the configuration of each pair $A_{n}, A_{n-1}$, with respect to the free boundary, is similar (after scaling) to that of $A_{1}, A_{0}$ (using the $\gamma$-flatness in each ball $B_{2^{-n}}\left(E_{n}\right)$ and the fact that the directions $\nu_{E_{n}}, \nu_{E_{n-1}}$ differ by at most $C \gamma / 2^{n}$ ), we also have, by Harnack's inequality, $u\left(A_{n}\right)>u\left(A_{n-1}\right) / N$ (with $N$ independent of $n$ ). Recalling (8.21) we obtain

$$
u\left(P_{n}\right) \geqslant \frac{1}{N^{n-1}} \frac{1}{\gamma^{n+1}} \frac{1}{N^{n-1}} u\left(A_{0}\right)=\frac{1}{\gamma^{2}} \frac{1}{\left(\gamma N^{2}\right)^{n-1}} u(A) .
$$

Choosing $\gamma<N^{2}$ we conclude that $u\left(P_{n}\right) \rightarrow \infty$ if $n \rightarrow \infty$, which is impossible. This completes the proof of (8.14).

Lemma 8.7 extends to $u^{-}$, that is, if $A_{*}=X_{0}-(0, \delta / 2)$ then

$$
u\left(A_{*}\right)<0, \quad u^{-}\left(A_{*}\right)>\gamma \sup _{B_{\delta / 2}\left(X_{0}\right)} u^{-} .
$$

COROLLARY 8.8. If $\gamma$ is small enough, say $\gamma<\gamma_{0}$, then

$$
\int_{B_{R}\left(X_{0}\right)}\left|\nabla u^{+}\right|^{2} \leqslant C\left(u^{+}(A)\right)^{2}, \quad \int_{B_{R}\left(X_{0}\right)}\left|\nabla u^{-}\right|^{2} \leqslant C\left(u^{-}\left(A_{*}\right)\right)^{2}
$$

where $C=C\left(\gamma_{0}\right)$ and $R=\delta\left(\gamma_{0}\right) / 4$. 
Indeed, introducing $G(X)=\log 2 R /\left|X-X_{0}\right|$ in $B_{2 R}\left(X_{0}\right)$, we have, by Green's formula,

$$
\begin{aligned}
-\int_{\partial B_{2 R}\left(X_{0}\right)}\left(u^{ \pm}\right)^{2} \frac{\partial G}{\partial \nu} & =\iint_{B_{2 R}\left(X_{0}\right)}\left[\Delta\left(u^{ \pm}\right)^{2} G-\left(u^{ \pm}\right)^{2} \Delta G\right] \\
& =2 \iint_{B_{2 R}\left(X_{0}\right)}\left|\nabla u^{ \pm}\right|^{2} G \geqslant c \iint_{B_{R}\left(X_{0}\right)}\left|\nabla u^{ \pm}\right|^{2},
\end{aligned}
$$

and the left-hand side is estimated by (8.14) and (8.22).

LEMMA 8.9. If $X_{0} \in \partial\{u>0\}$ and

$$
\underset{X \rightarrow X_{0}}{\limsup }\left|\nabla u^{-}(X)\right|=\alpha, \quad \limsup _{X \rightarrow X_{0}}\left|\nabla u^{+}(X)\right|=\beta,
$$

then

$$
\begin{aligned}
& \alpha^{2}\left(\alpha^{2}+|\Lambda|\right) \leqslant \frac{1}{R^{4}} \int_{B_{R}\left(X_{0}\right)}\left|\nabla u^{-}\right|^{2} \cdot \int_{B_{R}\left(X_{0}\right)}\left|\nabla u^{+}\right|^{2}, \\
& \beta^{2}\left(\beta^{2}-|\Lambda|\right) \leqslant \frac{1}{R^{4}} \int_{B_{R}\left(X_{0}\right)}\left|\nabla u^{-}\right|^{2} \cdot \int_{B_{R}\left(X_{0}\right)}\left|\nabla u^{+}\right|^{2}
\end{aligned}
$$

here $u$ is any minimizer in $B_{R}\left(X_{0}\right)$.

Proof. It suffices to prove (8.24). We take $X_{0}=0$ and $X_{n} \rightarrow 0$ with $\left|\nabla u^{-}\left(X_{n}\right)\right| \rightarrow \alpha$; we may suppose that $\alpha>0$. Let $Y_{n}$ be the nearest point to $X_{n}$ on the free boundary. Consider a blow-up sequence with respect to $B_{r_{n}}\left(Y_{n}\right), r_{n}=\left|X_{n}-Y_{n}\right|$. Since $\alpha>0$ and $\Lambda<0$, the blow-up limit has two phases and, by Lemma 8.3 , it is a 2-plane solution with slopes $\alpha$ and $\bar{\alpha}$ satisfying $\alpha^{2}-\bar{\alpha}^{2}=\Lambda$. It easily follows that, as $\varepsilon \rightarrow 0$,

$$
\frac{1}{\varepsilon^{2}} \int_{B_{\varepsilon}}\left|\nabla u^{-}\right|^{2} \rightarrow \alpha^{2}, \quad \frac{1}{\varepsilon^{2}} \int_{B_{\varepsilon}}\left|\nabla u^{+}\right|^{2} \rightarrow \bar{\alpha}^{2}
$$

The assertion (8.24) now follows using the monotonicity lemma.

LEMMA 8.10. Under the conditions of Corollary 8.8 (with $\left.\gamma<\gamma_{0}\right)$,

$$
\left|\nabla u^{-}(X)\right| \leqslant C u^{-}\left(A_{*}\right)\left(u^{+}(A)+1\right) \text { in } B_{R / 2}\left(X_{0}\right) \text {, }
$$

where $C$ is a constant (depending on $\gamma_{0}$ ).

Proof. The function $w=\left|\nabla u^{-}\right|$is subharmonic. By Lemma 8.9 and Corollary 8.8,

$$
\limsup _{X \rightarrow X_{0}} w(X) \leqslant C\left[u^{+}(A) u^{-}\left(A_{*}\right)\right]^{1 / 2}
$$

where $A=A\left(X_{0}\right), A_{*}=A_{*}\left(X_{0}\right)$. If $\gamma$ is small enough then by Harnack's inequality

$$
u^{+}\left(A\left(X_{0}\right)\right) \leqslant C u^{+}(A), \quad u^{-}\left(A_{*}\left(X_{0}\right)\right) \leqslant C u^{-}\left(A_{*}\right)
$$

where $A, A_{*}$ correspond to the free boundary point 0 and $X_{0} \in B_{R}$. Hence,

$$
\limsup _{X \in B_{R}, \operatorname{dist}(X, \partial\{u>0\}) \rightarrow 0} w(X) \leqslant C\left[u^{+}(A) u^{-}\left(A_{*}\right)\right]^{1 / 2} .
$$


On the other hand, by Corollary 8.8 and (8.27),

$$
\int_{B_{R} \cap\{u<0\}} w^{2} \leqslant C\left(u^{-}\left(A_{*}\right)\right)^{2} .
$$

Set $W=\max \left\{w, C\left[u^{+}(A) u^{-}\left(A_{*}\right)\right]^{1 / 2}\right\}$ in $B_{R}$. By (8.28), $W$ is a continuous subharmonic function and, therefore, by elliptic estimates,

$$
W^{2}(X) \leqslant C \int_{B_{R}} W^{2} \leqslant C u^{+}(A) u^{-}\left(A_{*}\right)+C\left(u^{-}\left(A_{*}\right)\right)^{2},
$$

and (8.26) follows.

LEMMA 8.11. Lemma 8.2 remains true without the assumption (ii).

Proof. It suffices to establish that

$$
\Delta_{h, l} u>0 \text { on } \partial I^{\delta}
$$

for all $h, l, u$. Suppose this is not true for a sequence $u_{m}$ with $X=X_{m}, h=h_{m}$, $l=l_{m}$. If the intervals $\tilde{l}_{m}:\left(X_{m}, X_{m}+h_{m} l_{m}\right)$ lie in $\left\{u_{m}>0\right\}$ then we can proceed as before. Indeed, the blow-up limit $w$ with respect to $B_{\delta_{m}^{1 / 2}}\left(X_{m}\right)$ (or $B_{r_{m}}\left(E_{i}\right), E_{i}$ depends on $m$ ) is either a 1-plane solution with $w \geqslant 0$ (since $\Lambda<0$ ) or a 2-plane solution and its free boundary is $\{y=0\}$ (here we use Remark 8.1); thus we get a contradiction as before.

If $\tilde{l}_{m}$ lies in $\left\{u_{m}<0\right\}$ and if a blow-up limit $w$ turns out to be a 1-plane solution with $w=0$ if $\{y<0\}$, we do not get a contradiction. In order to derive a contradiction we shall work with $U_{m}=u_{m} / u_{m}^{-}\left(A_{*}\right)$ instead of $u_{m}$, where $A_{*}$ is chosen as in Lemma $8.10\left(A_{*}\right.$ depends on $m$ ). Then $U_{m}\left(A_{*}\right)=-1$ and $U_{m}^{-}$is uniformly Lipschitz continuous (by Lemma (8.10)). Taking a blow-up limit $W$ of $U_{m}^{-}$ with respect to $B_{\delta_{m}^{1 / 2}}\left(X_{m}\right)$ (or $B_{r_{m}}\left(E_{i}\right)$ ) we find that the free boundary of $W$ is $\{y=0\}$; hence, by Liouville's theorem (reflecting first $W$ across $\{y=0\}$ ) $W \equiv c y$ if $y<0(c>0)$, and therefore, $\Delta_{h_{m}, l_{m}} U_{m} \geqslant c$ uniformly with respect to $\tilde{l}_{m}$ in $\left\{u_{m}<0\right\}$, that is,

$$
\Delta_{h_{m}, l_{m}} u_{m}>c u_{m}\left(A_{*}\right)>0
$$

uniformly with respect to $h_{m}, l_{m}, X_{m}$.

It remains to establish uniform positivity (in the sense of (8.30)) in case $\tilde{l}_{m}$ lies partially in $\left\{u_{m}>0\right\}$ and partially in $\left\{u_{m}<0\right\}$. In this case we can write it as a disjoint union of intervals $\tilde{l}_{m}=l_{m}^{1}+l_{m}^{2}+l_{m}^{3}$ where $l_{m}^{1} \subset\left\{u_{m}>0\right\}, l_{m}^{2} \subset\left\{u_{m}<0\right\}$ and $l_{m}^{3}$ is an interval with endpoints on $\sigma$ and $\sigma_{i}$. By Remark 8.1, meas $\left(l_{m}^{3}\right)=o\left(h_{m}\right)$ and thus either meas $\left(l_{m}^{1}\right)>c h_{m}$, or meas $\left(l_{m}^{2}\right)>c h$, or both inequalities hold. By the previous arguments for $\tilde{l}_{m}$ in $\left\{u_{m}>0\right\}$ and for $\tilde{l}_{m}$ in $\left\{u_{m}<0\right\}$ we deduce that the incremental quotients $\Delta_{l_{m}} u$ with respect to $l_{m}^{i}$ satisfy

$$
\begin{aligned}
& \Delta_{l_{m}^{\prime}} u \geqslant c \text { meas }\left(l_{m}^{1}\right) / \operatorname{meas}\left(\tilde{l}_{m}\right), \\
& \Delta_{l_{m}^{2}} u \geqslant c u_{m}\left(A_{*}\right) \operatorname{meas}\left(l_{m}^{2}\right) / \operatorname{meas}\left(\tilde{l}_{m}\right) .
\end{aligned}
$$

Since also $\Delta_{l_{m}^{3}} u \geqslant 0$, the assertion (8.29) holds. We can now proceed as in Lemma 8.2 to complete the proof of Lemma 8.11 . 
THEOREM 8.12. The free boundary $\partial\{u>0\} \cap \Omega$ is continuously differentiable.

Proof. By Lemma 8.5, for any small $\gamma>0$ there is a $\delta=\delta(\gamma)>0(\delta \downarrow 0$ if $\gamma \downarrow 0)$ such that the $\gamma$-flatness condition holds in every ball $B_{\delta}$ with center in the free boundary. Take such a ball $B_{\delta}$ and suppose for simplicity that its center is at the origin and that the flatness direction is $(0,1)$. By Lemma 8.11 the free boundary in $B_{\delta / 2}$ has the form $y=f(x)$ with $f(x)$ Lipschitz continuous.

Denote by $\gamma=\gamma(\delta)$ the inverse of the function $\delta=\delta(\gamma)$.

Take $x_{1}, x_{2}$ in $(-\delta / 4, \delta / 4)$ and set

$$
r=\left|x_{1}-x_{2}\right|, \quad X_{i}=\left(x_{i}, f\left(x_{i}\right)\right), \quad B_{i}=B_{2 r}\left(X_{i}\right) .
$$

Each $X_{i}$ must lie in the flatness strip of the disc $B_{j}(j \neq i)$. Therefore, the angles between the directions of flatness at $X_{1}$ and $X_{2}$ are bounded by $C \gamma(r)$. It follows that $\left|f^{\prime}\left(x_{1}\right)-f^{\prime}\left(x_{2}\right)\right| \leqslant C \gamma(r)$ for any two points $x_{1}, x_{2}$ where $f(x)$ is differentiable. Thus $f^{\prime}(x)$ has a continuous version.

The next result is concerned with the continuity of the normal derivative of $u$. Letting

$$
\gamma=\lim _{r \uparrow \infty} \frac{1}{r^{4}} \int_{B_{r}\left(X_{0}\right)}\left|\nabla u^{-}\right|^{2} \cdot \int_{B_{r}\left(X_{0}\right)}\left|\nabla u^{+}\right|^{2}
$$

where $X_{0}$ is a free boundary point, we define $\beta=\beta(\gamma)>0$ by $\beta^{2}\left(\beta^{2}-|\Lambda|\right)=\gamma$ and denote by $\nu=\nu_{X_{0}}$ the normal to the free boundary at $X_{0}$ (pointing into $\{u>0\})$.

THEOREM 8.13. For any sector

$$
\Sigma_{c}=\left\{X ;\left(X-X_{0}\right) \cdot \nu>c\left|X-X_{0}\right|\right\}, \quad c>0,
$$

there holds $u_{\nu}(X) \rightarrow \beta$ if $X \in \Sigma_{c}, X \rightarrow X_{0}$.

Proof. Let $X_{m} \in \Sigma_{c}, X_{m} \rightarrow X_{0}$ and take a blow-up sequence $u_{m}$ with respect to $B_{\left|X_{m}-X_{0}\right|}\left(X_{0}\right)$. Then $u_{m}(X) \rightarrow v(X)=\beta y(y>0)$ and $\partial u_{m}\left(X_{m}\right) / \partial \nu \rightarrow \partial v\left(Y_{0}\right) / \partial y$ since $Y_{0}$ lies in $\{y>0\}$. Since $\partial v\left(Y_{0}\right) / \partial y=\beta$, the assertion follows.

\section{REFERENCES}

1. H. W. Alt and L. A. Caffarelli, Existence and regularity for a minimum problem with free boundary, J. Reine Angew. Math. 325 (1981), 105-144.

2. H. W. Alt, L. A. Caffarelli and A. Friedman, Axially symmetric jet flows, Arch. Rational Mech. Anal. 81 (1983), 97-149.

3. Asymmetric jet flows, Comm. Pure Appl. Math. 35 (1982), 29-68.

4. Jet flows with gravity, J. Reine Angew. Math. 331 (1982), 58-103.

5. Jets with two fluids. I. One free boundary, Indiana Univ. Math. J. (to appear).

6. Jets with two fluids. II. Two free boundaries, Indiana Univ. Math. J. (to appear).

7. H. Federer, Geometric measure theory, Springer-Verlag, Berlin, Heidelberg and New York, 1969.

8. S. Friedland and W. K. Hayman, Eigenvalue inequalities for the Dirichlet problem on spheres and the growth of subharmonic functions, Comment. Math. Helv. 51 (1976), 133-161.

9. A. Friedman, Variational principles and free boundary problems, Interscience, New York, 1982.

10. E. Giusti, Minimal surfaces and functions of bounded variation, Notes on Pure Mathematics, Australian National Univ., Canberra, 1977. 
11. C. B. Morrey, Multiple integrals in the calculus of variations, Springer-Verlag, Berlin, Heidelberg and New York, 1966

12. E. Sperner, Zur symmetrisierung von funktionen auf sphären, Math. Z. 134 (1973), 317-327.

Institute fUr Angewandte Mathematik, Universität Bonn, D-5300 Bonn, Germany (Current address of $\mathrm{H}$. W. Alt)

Department of Mathematics, University of Chicago, Chicago, Illinois 60637

Department of Mathematics, Northwestern University, Evanston, Illinois 60201 (Current address of Avner Friedman)

Current address (L. A. Caffarelli): Department of Mathematics, University of Chicago, Chicago, Illinois 60637 